\title{
Spectral broadening of tonal sound propagating through an axisymmetric turbulent shear layer
}

\author{
Alan McAlpine* and Brian J. Tester ${ }^{\dagger}$ \\ Institute of Sound and Vibration Research, University of Southampton, Southampton, SO17 1BJ, United Kingdom
}

\begin{abstract}
In aeroacoustics, spectral broadening refers to the scattering of tonal sound fields by turbulent shear layers, whereby the interaction of the sound with turbulent flow results in power lost from the tone and distributed into a broadband field around the tone frequency. Fan and turbine tone spectral broadening is known colloquially as "haystacking”. Recently a new analytical model has been derived to predict weak spectral broadening of a tone radiated through a circular jet. A key part of the modeling is the choice of the two-point turbulent velocity cross-correlation function which is used to provide a statistical description of the turbulence in the shear layer. A new cross-correlation function for an axisymmetric turbulent shear layer formed by a circular jet, based on the theory for homogeneous axisymmetric turbulence, has been developed. Validation results of weak-scattering calculated using this correlation function show better agreement with measurements when compared to the results calculated using a correlation function based on the theory for homogeneous isotropic turbulence.
\end{abstract}

\section{Nomenclature}

$$
\begin{array}{ll}
c & =\text { speed of sound }\left[\mathrm{ms}^{-1}\right] \\
C_{p} & =\text { specific heat capacity at constant pressure }\left[\mathrm{Jkg}^{-1} \mathrm{~K}^{-1}\right] \\
C_{V} & =\text { specific heat capacity at constant volume }\left[\mathrm{Jkg}^{-1} \mathrm{~K}^{-1}\right] \\
d & =\text { Doppler factor, incident field }[\mathrm{Hz}] \\
D & =\text { Doppler factor, scattered field }[\mathrm{Hz}] \\
\mathbf{e}^{(1)}, \mathbf{e}^{(2)} & =\text { unit vectors defining orthogonal coordinate system } \\
f & =\text { frequency, incident field }[\mathrm{Hz}] \\
F & =\text { frequency, scattered field }[\mathrm{Hz}]
\end{array}
$$

\footnotetext{
*Associate Professor, Institute of Sound and Vibration Research, University of Southampton.

†Principal Research Fellow, Institute of Sound and Vibration Research, University of Southampton. AIAA Senior member.
} 


$$
\begin{array}{ll}
k & =\text { axial wavenumber, incident field }\left[\mathrm{radm}^{-1}\right] \\
K & =\text { axial wavenumber, scattered field }\left[\mathrm{radm}^{-1}\right] \\
\mathcal{L} & =\text { integral lengthscale }[\mathrm{m}] \\
m & =\text { azimuthal order, incident field } \\
M & =\text { azimuthal order, scattered field } \\
p & =\text { pressure }[\mathrm{Pa}] \\
r_{c} & =\text { jet radius [m] } \\
\mathbf{r} & =\text { separation vector }[\mathrm{m}] \\
R & =\text { specific gas constant }\left[\mathrm{Jkg}^{-1} \mathrm{~K}^{-1}\right] \\
R_{i j} & =\text { turbulent velocity two-point cross-correlation } \\
\mathcal{R}_{i j} & =\text { turbulent velocity two-point cross-correlation (without temporal decay) } \\
s & =\text { azimuthal separation } \bar{r} \mu_{\phi}[\mathrm{m}] \\
t & =\text { time [s] } \\
T & =\text { temperature }[\mathrm{K}] \\
\mathcal{T} & =\text { integral timescale }[\mathrm{s}] \\
\mathbf{u} & =\text { velocity }(u, v, w)\left[\mathrm{ms}{ }^{-1}\right] \\
U_{c} & =\text { turbulence convection velocity }\left[\mathrm{ms}^{-1}\right] \\
U_{J} & =\text { core jet flow velocity }\left[\mathrm{ms}^{-1}\right] \\
U(r) & =\text { mean velocity profile }\left[\mathrm{ms}^{-1}\right] \\
(x, r, \phi) & =\text { polar coordinate system } \\
(x, y, z) & =\text { Cartesian coordinate system } \\
\mathcal{L} &
\end{array}
$$

Greek variables

$$
\begin{array}{ll}
\beta & =\text { non-dimensional constant used to define temporal decay function } \\
\gamma & =\text { adiabatic constant } C_{p} / C_{V} \\
\gamma_{m} & =\text { radial wavenumber, incident field }\left[\mathrm{radm}^{-1}\right] \\
\Gamma_{M} & =\text { radial wavenumber, scattered field }\left[\mathrm{radm}^{-1}\right] \\
\delta & =\text { shear layer thickness }[\mathrm{m}] \\
\theta & =\text { polar angle, incident field }[\mathrm{rad}] \\
\Theta & =\text { polar angle, scattered field [rad] }
\end{array}
$$




$$
\begin{array}{ll}
\lambda & =\text { unit vector defining axis of symmetry } \\
\mu & =\text { spatial separation distance }[\mathrm{m}] \\
\rho & =\text { density }\left[\mathrm{kgm}^{-3}\right] \\
\tau & =\text { temporal separation time }[\mathrm{s}] \\
\Phi_{i j} & =\text { turbulent velocity cross-spectrum } \\
\omega & =\text { angular frequency, incident field }\left[\mathrm{rads}^{-1}\right] \\
\Omega & =\text { angular frequency, scattered field }\left[\mathrm{rads}^{-1}\right]
\end{array}
$$

\section{Subscripts}

$d \quad=$ denotes directivity of the incident field

$D \quad=$ denotes directivity of the scattered field

$i, j \quad=\quad$ indices denoting $x, r$ or $\phi$

$m \quad=$ denotes azimuthal order of the incident field

$M \quad=$ denotes azimuthal order of the scattered field

$s \quad=\quad$ denotes sound (acoustic) quantity

$t=$ denotes turbulent quantity

$\infty \quad=$ denotes value at infinity

Superscripts

, = denotes perturbation quantity

Symbols

$\rightarrow \quad=$ denotes moving reference frame

- $\quad=\quad$ denotes mean quantity

$\sim \quad=$ denotes the Fourier transform of a quantity

- $\quad=$ denotes a normalised quantity

\langle\rangle$\quad=$ denotes ensemble average

\section{Introduction}

$\mathrm{T}^{\mathrm{N}}$ the past, aircraft engine noise sources such as jet noise were dominant, but owing to significant progress in the reduction of aircraft noise, in modern engines a wider range of noise sources contribute appreciable amounts to the overall noise spectrum. This means that sources which previously had received less attention, such as core and turbine 
noise, are becoming more important.

Spectral broadening in aeroacoustics is caused when sound propagates through a turbulent flow. Specifically it usually relates to scattering of tonal fields whereby the acoustic energy in the tone is scattered into adjacent frequencies, owing to interaction between the sound field and the turbulent flow. The scattering is "weak" if the tone remains prominent in the frequency spectrum, that is only a small proportion of the energy in the tone is scattered into neighboring frequency bands. However the scattering can be "strong", which is when the original tone after the scattering is no longer prominent in the spectrum. Owing to a large proportion of the energy being scattered, this typically leads to the appearance of a broadband "hump" in the spectrum.

This effect is prominent for turbine tones, and to a lesser extent fan tones, radiated from the exhaust of a turbofan jet engine, see Fig. 1 Turbine-tone spectral broadening is known colloquially as "haystacking". Spectral broadening causes substantial difficulties in determining the relative importance of various sources of turbofan engine noise, as it scatters sound generated by tonal sources across a wide frequency range. This can pose a serious challenge when interpreting measurements of engine noise. For example, if turbine tones undergo strong scattering, the tones may not be detected in measurements obtained outside the jet.

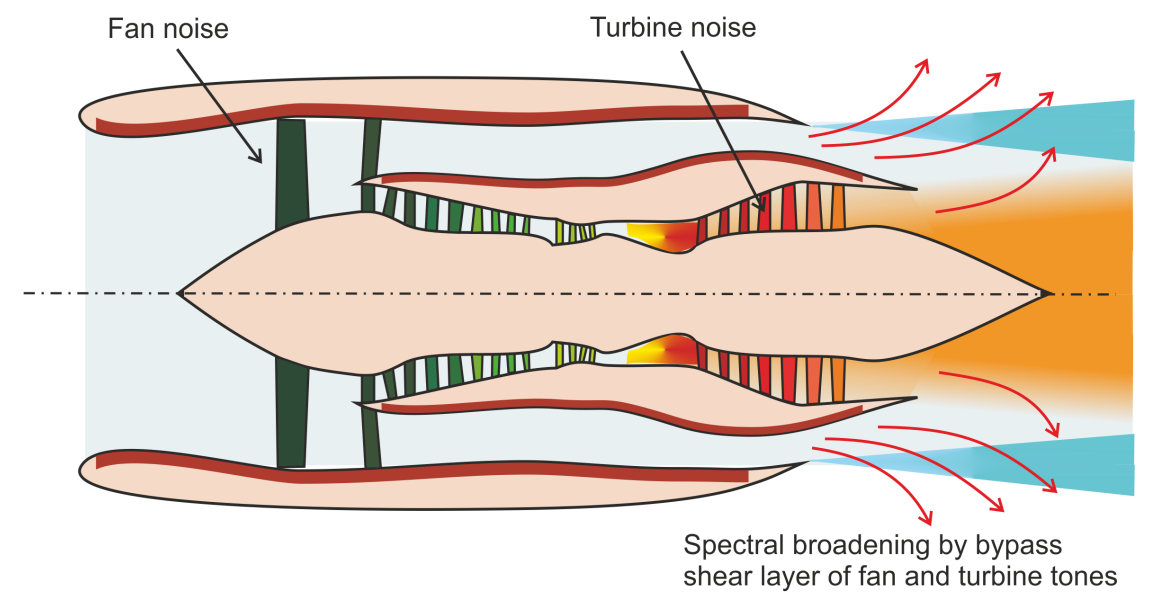

Fig. 1 Spectral broadening of tonal sound fields by jet shear layers - turbofan engine application.

There is only a small body of published work in aeroacoustics on the topic of spectral broadening. Candel, Guedel \& Julienne [1] report laboratory experiments on model-scale jets. In these experiments a point source is situated inside a circular jet, and a microphone is situated outside. Their measurements provide examples of weak spectral broadening at polar angle equal to $90^{\circ}$. 
Ahuja, Tester and Tanna [2] conducted a major study on flight effects on jet noise. This included experimental and theoretical analysis of the effect of sound transmission through a shear layer, to understand the differences between flight data and measurements in a free-jet that will be affected by reflection, refraction and turbulence scattering leading to spectral broadening.

Campos [3, 4] published a theoretical study on tone spectral broadening. His model incorporates a number of important effects, including source directivity. The turbulence scattering is modeled by a phase-screen approach; the turbulence is assumed to create a random phase modulation of the field.

Guédel [5] also examined the experimental results from Candel et al. [1] and compared measurements against a theoretical analysis based on a single scattering method. Furthermore they estimated the ratio of the scattered energy to the total energy.

More recently a new analytical model to predict weak spectral broadening of a tone radiated through a circular jet has been derived by the authors. Full details of this solution are published in Powles, Tester \& McAlpine [6] and McAlpine, Powles \& Tester [7]; brief details also are provided in Sec. [I] This solution is based on an approach originally developed by the late Alexander M. Cargill (Rolls-Royce plc). In 1983 Cargill reviewed, in the context of aeroacoustics, scattering of sound by unsteady flows [8]. Subsequently, in 1989, he developed a theoretical model of sound scattering by a turbulent plane shear layer, although this work is not publicly available.

Additionally, numerical simulations of weak scattering have been demonstrated in a number of recent studies. Ewert and co-authors [9, 10] have modeled weak spectral broadening using an in-house computational aeroacoustics code called PIANO developed at the German Aerospace Center (DLR). The method is based on solving the linearised Euler equations in the time domain using a high-order finite difference scheme. The unsteady turbulent base flow is modeled using a stochastic method to generate turbulence features provided by time-averaged RANS calculations. The PIANO code also was used by Clair and Gabard [11,12] who studied acoustic wave scattering by a single, convected vortex to investigate the physical mechanisms of spectral broadening by turbulence. They showed that for a single vortex, sidebands in the spectrum at frequencies above and below the tone are generated by a combination of spatial scattering by refraction, and two Doppler shifts owing to the relative motions between the vortex, source and observer. Subsequently Clair and Gabard [13] carried out a numerical investigation of spectral broadening for radiation from a monopole source scattered by a layer of turbulence convected by a uniform flow. Furthermore, Bennaceur et al. [14] carried out the first study of spectral broadening using the Large Eddy Simulation (LES) method. They compared their numerical results against measurements from Candel, Guedel \& Julienne [1] and similar measurements from wind 
tunnel tests by Kröber, Hellmond \& Koop [15].

Although the early measurements of spectral broadening by Candel, Guedel \& Julienne [1] have been the most cited for validation studies, a collection of more recent measurements have been compiled by Sijtsma et al. [16]. Furthermore, Sijtsma et al. derived a simple model, referred to as the " $\beta$-correlation", based on the effect of time-delay variations to predict the ratio of the acoustic energy which is scattered out of the tone, and in to the adjacent broadband "haystack". (Guédel [5] also derived an expression for this ratio.) This semi-empirical method was then validated against the collection of wind-tunnel spectral broadening measurements in Ref. [16]. Since the " $\beta$-correlation" validation results were rather mixed, subsequently Tester \& Sijtsma [17] used beamforming on a dataset acquired previously at the QinetiQ Noise Test Facility, in order to better understand the source generation mechanism of spectral broadening.

In this article the aim is to use the analytical model for weak scattering from Refs. [6, 7] to simulate spectral broadening for validation test cases based on the measurements from Candel, Guedel \& Julienne [1]:* A key aspect of the modelling is the choice of the two-point turbulent velocity cross-correlation function which is used to provide a statistical description of the turbulence in the shear layer. The objective of this current work is to examine three different cross-correlation functions. The first cross-correlation is based on a standard Gaussian function. The second cross-correlation function is derived based on the theory of three-dimensional homogeneous isotropic turbulence, and was originally proposed in Refs. [6, 7]. The third cross-correlation function, and the key result in this article, is a new formulation derived based on the theory of homogeneous axisymmetric turbulence, which can be used for the turbulent shear layer formed by a circular jet. In this article, simulation results employing different turbulent velocity cross-spectra, obtained by taking the Fourier transform of the three different cross-correlation functions, are compared.

This article is organised as follows. Brief details of the weak scattering method from Refs. [6, 7] are reproduced in Sec. III. Formulations for the different turbulent velocity cross-correlation functions and spectra are detailed in Sec. III] Simulation results are shown in Sec. IV Finally, the results are discussed in Sec. V, and the key conclusions are summarised in Sec.VI.

\section{Weak-scattering model}

The derivation of the weak-scattering model is in Powles, Tester \& McAlpine [6] and McAlpine, Powles \& Tester [7]. In this section brief details are reproduced from Ref. [7]

\footnotetext{
* Some of the material in this article was originally presented at the 22nd AIAA/CEAS Aeroacoustics conference, see reference [18].

$\dagger$ In the mathematical analysis a slightly unconventional notation is used. Vector quantities are denoted in bold, whereas an arrow denotes a variable in a moving reference frame not a vector.
} 
In the modelling, the propagation of sound through an axisymmetric turbulent shear layer is considered. The shear layer is formed by a circular jet exiting into quiescent fluid. The coordinate systems are shown in Fig. 22(a). The turbulence is largely confined to the region where there is shear flow. At any arbitrary axial station, taking a cross-sectional slice through the jet would reveal the turbulence spread over an annular disc, as shown in Fig. 2(b). The velocity of the core jet flow is $U_{J}$. Outside the jet, the velocity is zero. The centre of the turbulent shear layer, $r=r_{c}$, is the radial position where the velocity $U$ equals $\frac{1}{2} U_{J}$. This is referred to as the jet radius. The thickness of the shear layer is denoted by $\delta$.

The fluid flow is expressed as the sum of a steady mean flow and a small unsteady fluctuating component. The mean flow is assumed to be parallel and axisymmetric, hence

$$
\overline{\mathbf{u}}=\left(\bar{u}_{x}, \bar{u}_{r}, \bar{u}_{\phi}\right)=(U(r), 0,0)
$$

The mean flow pressure, density and temperature profiles are expressed in the form

$$
\bar{p}=\text { constant }, \bar{\rho}=\bar{\rho}(r) \text { and } \bar{T}=\bar{T}(r),
$$

respectively. For an isentropic perfect gas absolute temperature and speed of sound are related by $c^{2}=\gamma R T$, where $\gamma=C_{p} / C_{V}$ is the ratio of specific heat capacities, and $R$ is the specific gas constant. Hence the mean square sound speed profile, $\overline{c^{2}}(r)$, is used in place of the temperature profile $\bar{T}(r)$.

The small unsteady perturbations are given by

$$
\mathbf{u}^{\prime}=\mathbf{u}-\overline{\mathbf{u}}, \quad p^{\prime}=p-\bar{p}, \quad \rho^{\prime}=\rho-\bar{\rho} \text { and } \quad c^{2^{\prime}}=c^{2}-\overline{c^{2}} .
$$

An approximate form of the Lilley equation is used to model the scattering of sound by turbulence. It is expressed in the form

$$
L\left[p^{\prime}\right]=S,
$$

where the Pridmore-Brown operator

$$
L=\left[\frac{\overline{\mathrm{D}}}{\mathrm{D} t}\left(\frac{\overline{\mathrm{D}}^{2}}{\mathrm{D} t^{2}}-\nabla \cdot\left(\overline{c^{2}} \nabla\right)\right)+2 \overline{c^{2}}\left(\frac{\mathrm{d} U}{\mathrm{~d} r}\right) \frac{\partial^{2}}{\partial x \partial r}\right], \quad \frac{\overline{\mathrm{D}}}{\mathrm{D} t}=\frac{\partial}{\partial t}+U \frac{\partial}{\partial x},
$$




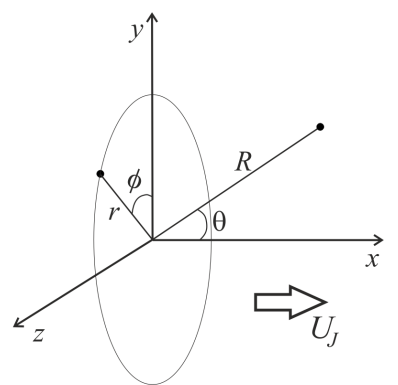

(a)

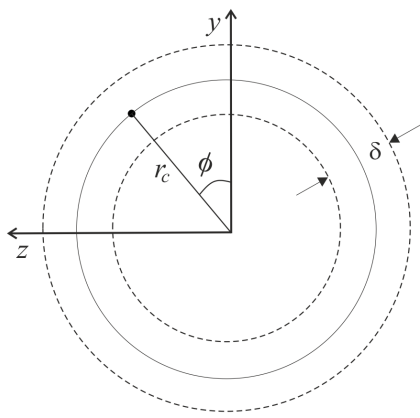

(b)

Fig. 2 Circular jet centred on the $x$-axis, jet velocity $U_{J}$ • (a) Cylindrical polar coordinates $(x, r, \phi)$ and spherical polar coordinates $(R, \theta, \phi)$. (b) Cross-section of the turbulent shear layer formed by the circular jet. The jet radius is $r_{c}$, and the turbulent shear layer thickness is $\delta$. 
and the source term for an unheated jet

$$
S=\bar{\rho} \overline{c^{2}} \frac{\overline{\mathrm{D}}}{\mathrm{D} t}\left[\frac{\partial^{2}}{\partial x_{i} \partial x_{j}}\left\{u_{i}^{\prime} u_{j}^{\prime}\right\}\right]
$$

using Cartesian tensor notation. Equation (4) is an approximate version of an exact form of Lilley's equation derived by Goldstein [19]. The key modification is a high-frequency approximation, whereby source terms which involve the gradient of a mean flow quantity have been omitted in Eq. 6.

The velocity perturbation $\mathbf{u}^{\prime}$ is described as the sum of a turbulent and acoustic field, i.e. $u_{i}^{\prime}=u_{i t}^{\prime}+u_{i s}^{\prime}$, where subscript $t$ denotes turbulence and subscript $s$ denotes sound. In the source term (6) only turbulent/acoustic field interactions are retained, so

$$
u_{i}^{\prime} u_{j}^{\prime} \approx u_{i t}^{\prime} u_{j_{s}}^{\prime}+u_{i s}^{\prime} u_{j_{t}}^{\prime}
$$

The total pressure perturbation $p^{\prime}$ is split into two parts, termed the "incident" and "scattered" fields. The incident field is defined as the field that would be present in the absence of any scattering, i.e. in the absence of turbulence. The scattered field is the field due to the interaction of the turbulence with the incident field.

In the analysis lower-case variables are used to denote incident field quantities and upper-case variables are used to denote scattered field quantities. This necessitates a slight change in notation. The pressure perturbation is expressed as $p^{\prime}+P^{\prime}$, where $p^{\prime}$ denotes the incident field and $P^{\prime}$ denotes the scattered field. In the case of weak scattering $\left|P^{\prime}\right|<<\left|p^{\prime}\right|$.

Fourier transforms and high-frequency asymptotics are used to solve the problem. Denoting transformed variables

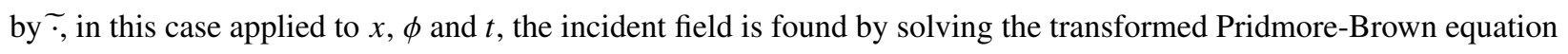

$$
\widetilde{L}\left[\widetilde{p^{\prime}}\right]=0
$$

and the scattered field is found by solving the transformed Lilley equation (4)

$$
\widetilde{L}\left[\widetilde{P}^{\prime}\right]=\widetilde{S}
$$

The solution of the transformed Lilley equation (9) is found in terms of a Green function integral equation. The weak scattering assumption means that the acoustic velocity perturbation terms $u_{i s}^{\prime}$ are taken to be the incident acoustic field only, and can be expressed in terms of the incident pressure field $p^{\prime}$ which is given by the solution of the transformed Pridmore-Brown equation (8). It is then convenient to form the spectral density because this means that the description 
of the turbulence will be required in terms of a turbulent velocity spectrum. Finally, this is linked to the far-field directivities of the incident and scattered fields which are expressed in spherical polar coordinates $(R, \theta, \phi)$. The key result of the analysis is

$$
\widetilde{P}_{M_{D}}(\Theta, \Omega) \sim \frac{1}{(2 \pi)^{3}}\left|K_{\infty}\right| \sin \Theta \sum_{m=-\infty}^{\infty} \int_{\omega=-\infty}^{\infty} \int_{\theta=0}^{\pi} \widetilde{p}_{m_{d}}(\theta, \omega) \mathcal{H}\left(\left|K_{\infty}\right| \cos \Theta, M, \Omega,\left|k_{\infty}\right| \cos \theta, m, \omega\right) \mathrm{d} \omega \mathrm{d} \theta
$$

The far-field mean square pressure directivity of the scattered pressure field $\widetilde{P}_{M_{D}}(\Theta, \Omega)$, at polar angle $\Theta$ and frequency $\Omega$, is defined by $\lim _{R \rightarrow \infty} R^{2}\left(\frac{1}{2}\left|\widetilde{P}^{\prime}{ }_{M}(R, \Theta, \Omega)\right|^{2}\right)$ which represents the $M$ th azimuthal order to the circumferentially averaged sound field. The far-field mean square pressure of the incident pressure field $\widetilde{p}_{m_{d}}(\theta, \omega)$ at polar angle $\theta$ and frequency $\omega$ is defined similarly. From Eq. (10) the far-field mean square pressure directivity of the scattered pressure field $\widetilde{P}_{M_{D}}(\Theta, \Omega)$ is specified in terms of a summation over azimuthal order $m$, and integrations over frequency $\omega$, and polar angle $\theta$, of the product of the far-field mean square pressure directivity of the incident pressure field, $\widetilde{p}_{m_{d}}(\theta, \omega)$, multiplied by the function $\mathcal{H}$.

The function $\mathcal{H}$ may be viewed as a transfer function which relates the incident and scattered fields. It depends on $K=\left|K_{\infty}\right| \cos \Theta=\left(\Omega / c_{\infty}\right) \cos \Theta, M, \Omega, k=\left|k_{\infty}\right| \cos \theta=\left(\omega / c_{\infty}\right) \cos \theta, m$ and $\omega$. Here $K$ and $k$ denote the axial wavenumbers of the scattered and incident sound fields, respectively, and $c_{\infty}$ is the speed of sound. Also closely associated with these parameters are $\Gamma_{M}$ and $\gamma_{m}$. These denote the effective radial wavenumbers of the scattered and incident sound fields:

$$
\begin{aligned}
\Gamma_{M}^{2} & =\frac{D^{2}}{\overline{c^{2}}}-K^{2}-\frac{M^{2}}{r^{2}}, \\
\gamma_{m}^{2} & =\frac{d^{2}}{\overline{c^{2}}}-k^{2}-\frac{m^{2}}{r^{2}},
\end{aligned}
$$

where $D=(\Omega-K U)$ and $d=(\omega-k U)$ are Doppler factors.

In Refs. [6, 7] it is shown that $\mathcal{H}$ can be expressed directly in terms of the turbulent velocity cross-spectrum,

$$
\begin{aligned}
\Phi_{i j}\left(\bar{r}, K-k, \Gamma_{M}-\gamma_{m}, M-m, \Omega-\omega\right)=\int_{\mu_{x}=-\infty}^{\infty} \int_{\mu_{r}=-\infty}^{\infty} \int_{\mu_{\phi}=-\pi}^{\pi} \int_{\tau=-\infty}^{\infty} \\
R_{i j}\left(\bar{r}, \mu_{x}, \mu_{r}, \mu_{\phi}, \tau\right) e^{\mathrm{i}(K-k) \mu_{x}} e^{\mathrm{i}\left(\Gamma_{M}-\gamma_{m}\right) \mu_{r}} e^{\mathrm{i}(M-m) \mu_{\phi}} e^{-\mathrm{i}(\Omega-\omega) \tau} \mathrm{d} \tau \mathrm{d} \mu_{\phi} \mathrm{d} \mu_{r} \mathrm{~d} \mu_{x},
\end{aligned}
$$


which is found by taking the Fourier transform of the turbulent velocity cross-correlation function

$$
R_{i j}=\left\langle u_{i t}^{\prime}\left(x_{1}, r_{1}, \phi_{1}, t_{1}\right) u_{j_{t}}^{\prime}\left(x_{2}, r_{2}, \phi_{2}, t_{2}\right)\right\rangle
$$

where \langle\rangle denotes ensemble average. Since scattering by a circular jet is being considered, the velocity components are in the axial, radial and azimuthal directions, so it is understood that the subscripts $i$ and $j$ denote $x, r$ or $\phi$.

The mean flow is steady, parallel, and axisymmetric, hence it is assumed that the turbulence is statistically stationary in the variables $x, \phi$ and $t$. Then $R_{i j}$ is a function of only

$$
\bar{r}=\frac{1}{2}\left(r_{1}+r_{2}\right), \mu_{x}=x_{1}-x_{2}, \mu_{r}=r_{1}-r_{2}, s=\bar{r} \mu_{\phi}=\bar{r}\left(\phi_{1}-\phi_{2}\right) \text { and } \tau=t_{1}-t_{2},
$$

where $\bar{r}$ is the average radial position, $\mu$ denotes spatial separation variables, and $\tau$ denotes the temporal separation.

The full transfer function $\mathcal{H}$ is given by equation (82) in McAlpine, Powles \& Tester [7] - the derivation of the transfer function was the key result in Ref. [7]. In the current article, the simulation results in Sec. IV are at $\Theta=90^{\circ}$. Therefore, in the current article a simpler version of the transfer function, valid outside the cone of silence, is used. This is given by,

$$
\begin{aligned}
\mathcal{H}(K, M, \Omega, k, m, \omega)= & \int_{\bar{r}=0}^{\infty} \frac{2}{\bar{D}^{2}}\left(\frac{D_{\infty}}{d_{\infty}}\right)^{2}\left(\frac{\gamma_{m_{\infty}}}{\Gamma_{M_{\infty}}}\right)\left(\frac{1}{\bar{\gamma}_{m}^{2}}\right)^{\frac{1}{2}}\left(\frac{1}{\bar{\Gamma}_{M}^{2}}\right)^{\frac{1}{2}}\left(K k+\bar{\Gamma}_{M} \bar{\gamma}_{m}+\frac{M m}{\bar{r}^{2}}\right)^{2} \times \\
& {\left[K^{2} \Phi_{x x}+\bar{\Gamma}_{M}^{2} \Phi_{r r}+\left(\frac{M}{\bar{r}}\right)^{2} \Phi_{\phi \phi}+2 K \bar{\Gamma}_{M} \Phi_{x r}+2 K\left(\frac{M}{\bar{r}}\right) \Phi_{x \phi}+2 \bar{\Gamma}_{M}\left(\frac{M}{\bar{r}}\right) \Phi_{r \phi}\right] \mathrm{d} \bar{r}, }
\end{aligned}
$$

from equation (D.1) in Ref. [7]. The integration over $\bar{r}$ in Eq. 16] need only be evaluated over a finite range which spans the shear layer, outside this range the turbulent velocity cross-correlations will be negligible.

The formulation gives an expression for the far-field mean square pressure of the scattered field, valid for weak scattering. It can be shown that the spectral shape of the haystacked tone will be dependent on the shape of the turbulence spectrum $\Phi_{i j}$. The formulation is general, it is independent of the form of $\Phi_{i j}$. Consequently an appropriate choice of the turbulent velocity cross-correlation function $R_{i j}$, and its corresponding turbulent velocity cross-spectrum $\Phi_{i j}$, will be crucial to generate realistic simulations of spectral broadening. 


\section{Models of the turbulent velocity cross-correlation and cross-spectrum}

\section{A. The circular jet}

The turbulence in the shear layer formed by the circular jet is assumed to be statistically axisymmetric. Fully axisymmetric turbulence is defined as follows. The flow is rotationally symmetric, so the rate-of-change with respect to $\phi$, i.e. $\partial / \partial \phi$, of any statistical quantity associated with the turbulence equals zero. Also the turbulence is invariant under reflections in the azimuthal coordinate $\phi$. Thus the Reynolds stress for a circular axisymmetric jet is

$$
-\rho\left[\begin{array}{ccc}
\left\langle u_{x t}^{\prime 2}\right\rangle & \left\langle u_{x t}^{\prime} u_{r t}^{\prime}\right\rangle & 0 \\
\left\langle u_{x t}^{\prime} u_{r t}^{\prime}\right\rangle & \left\langle u_{r t}^{\prime 2}\right\rangle & 0 \\
0 & 0 & \left\langle u_{\phi_{t}}^{\prime 2}\right\rangle
\end{array}\right] .
$$

The flow in an axisymmetric turbulent circular jet is statistically two-dimensional. The statistical properties of the flow depend only on $x$ and $r$, they are independent of $\phi$. Experimental measurements of $\left\langle u_{x t}^{\prime 2}\right\rangle,\left\langle u_{r t}^{\prime 2}\right\rangle,\left\langle u_{\phi_{t}}^{\prime 2}\right\rangle$ and $\left\langle u_{x t}^{\prime} u_{r t}^{\prime}\right\rangle$ measured transversely across a top-hat turbulent jet flow with Reynolds number $10^{5}$ have been published by Hussein, Capp \& George [20]. It is seen that the quantities $\left\langle u_{r t}^{\prime 2}\right\rangle$ and $\left\langle u_{\phi_{t}}^{\prime 2}\right\rangle$ are comparable in magnitude but less compared with $\left\langle u_{x t}^{\prime 2}\right\rangle$. Also measurements of the quantity $\left\langle u_{x t}^{\prime} u_{r t}^{\prime}\right\rangle$ reveal that the magnitude is much lower compared to the isotropic elements of the Reynolds stress tensor.

\section{B. Two-point turbulence statistics and the Gaussian correlation function}

For a circular jet, from Eq. [12], an appropriate form of the two-point space-time turbulent velocity cross-correlation function, $R_{i j}$, is given by

$$
R_{i j}=\left\langle u_{t i}^{\prime} u_{t j}^{\prime}\right\rangle(\bar{r}) \widehat{R}_{i j}\left(\mu_{x}, \mu_{r}, s, \tau\right)
$$

where the function $\widehat{R}_{i j}$ is the normalised cross-correlation function. From this, the classic longitudinal (axial) turbulence integral length scale $\mathcal{L}$ and timescale $\mathcal{T}$ are defined as

$$
\begin{aligned}
\mathcal{L} & =\int_{0}^{\infty} \widehat{R}_{x x}\left(\mu_{x}, 0,0,0\right) \mathrm{d} \mu_{x}, \\
\mathcal{T} & =\int_{0}^{\infty} \widehat{R}_{x x}(0,0,0, \tau) \mathrm{d} \tau .
\end{aligned}
$$


Additionally, in the case of jet flow, the turbulence is convected. The turbulence convection velocity $U_{c}$ is a measure of the velocity at which the turbulent eddies propagate. What is now known as Taylor's hypothesis pertains to "frozen" turbulence. On assuming that the turbulence is frozen, the effect of the mean flow will be purely convective. In this case, $U_{c}=\mathcal{L} / \mathcal{T}$.

Turbulent velocity cross-correlation functions are formulated in terms of two-point turbulence statistical quantities which can be measured or predicted. The relevant quantities are the turbulence kinetic energy, turbulence convection velocity, and turbulence integral length and time scales. Perhaps the most commonly used form for the two-point turbulent velocity cross-correlation is based on the Gaussian function, for example Tam and Auriault [21] and Ewert [22]. For frozen turbulence, Refs. [21, 22] propose that an appropriate form of the longitudinal cross-correlation function is

$$
\widehat{R}_{x x}=\exp \left\{-\frac{\pi}{4}\left(\frac{\left[\mu_{x}-U_{c} \tau\right]^{2}+\mu_{r}^{2}+s^{2}}{\mathcal{L}^{2}}\right)\right\}
$$

Note that a factor of $\pi / 4$ is included in the exponent, because then the characteristic lengthscale in Eq. (21) is equivalent to the integral lengthscale $\mathcal{L}$.

In the case of non-frozen turbulence, a temporal function, say $\mathcal{F}(\tau)$, is included to model the time decay. Therefore, the normalised cross-correlation function is expressed in the general form by

$$
\widehat{R}_{i j}\left(\mu_{x}, \mu_{r}, s, \tau\right)=\mathcal{F}(\tau) \mathcal{R}_{i j}\left(\vec{\mu}_{x}, \mu_{r}, s\right)
$$

where $\vec{\mu}_{x}=\vec{x}_{1}-\vec{x}_{2}$, and $\vec{x}=x-U_{c} t$ is a moving frame of reference.

For frozen turbulence set $\mathcal{F}(\tau)=1$. For non-frozen turbulence, Morris and Boluriaan [23], and also Ewert [22], propose that an appropriate form of $\mathcal{F}(\tau)$ is given by

$$
\mathcal{F}(\tau)=\exp \{-\beta|\tau| / \mathcal{T}\}, \quad \beta>0
$$

Determination of the value of the non-dimensional constant $\beta$ is outlined in the Appendix.

Although the Gaussian correlation function is often used to describe turbulent flows, the function does not have a cusp at the origin which is observed from experimental measurements. In Refs. [6, 7], a modified Gaussian correlation function was proposed which was formulated using the established theory for homogeneous isotropic turbulence [24]. The formulation is briefly outlined in the next section. Then, in the following section, the formulation is extended to the 
case of homogeneous axisymmetric turbulence.

\section{Homogeneous Isotropic Turbulence}

A homogeneous isotropic turbulence field is one which is statistically homogeneous and statistically invariant under any reflection or rotation of the coordinate system. Batchelor [24] (§ 3.4) outlines the general form of the two-point space cross-correlation function for homogeneous isotropic turbulence.

Using the general form of an isotropic second-order tensor which is a function of $\mathbf{r}$ only,

$$
\widehat{R}_{i j}(\mathbf{r})=F(r) r_{i} r_{j}+G(r) \delta_{i j},
$$

where $F$ and $G$ are scalar functions of the separation distance $r=|\mathbf{r}|$, and $\delta_{i j}$ is the Kronecker-delta function.

Following Batchelor, set

$$
F(r)=\left(\frac{f(r)-g(r)}{r^{2}}\right) \quad \text { and } \quad G(r)=g(r)
$$

Batchelor shows that the functions $f(r)$ and $g(r)$ can be interpreted as the normalised longitudinal and lateral two-point velocity cross-correlation functions. An example is shown in Fig. 3 for the two-dimensional problem $(\vec{x}, r)$. Two different separation vectors are illustrated: (a) axial separation, $\vec{\mu}_{x}$; (b) radial separation, $\mu_{r}$. In case (a), the longitudinal function is $\widehat{R}_{x x}$ and the lateral function is $\widehat{R}_{r r}$, and vice versa in case (b).

Combining Eqs. 24] and 25] gives,

$$
\widehat{R}_{i j}(\mathbf{r})=\left[\frac{f(r)-g(r)}{r^{2}} r_{i} r_{j}+g(r) \delta_{i j}\right]
$$

Owing to the solenoidal nature of the turbulence field,

$$
\frac{\partial \widehat{R}_{i j}}{\partial r_{i}}=\frac{\partial \widehat{R}_{i j}}{\partial r_{j}}=0,
$$

which means that

$$
\frac{\partial}{\partial r_{i}}\left[\frac{f(r)-g(r)}{r^{2}} r_{i} r_{j}+g(r) \delta_{i j}\right]=0 .
$$




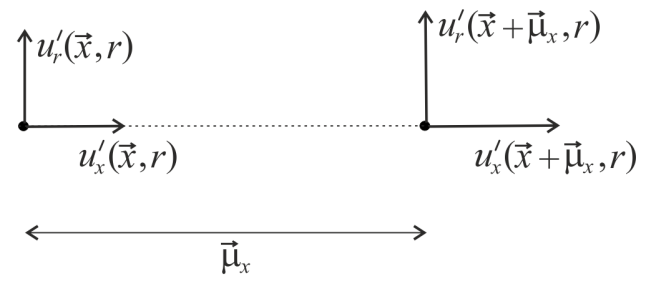

(a)

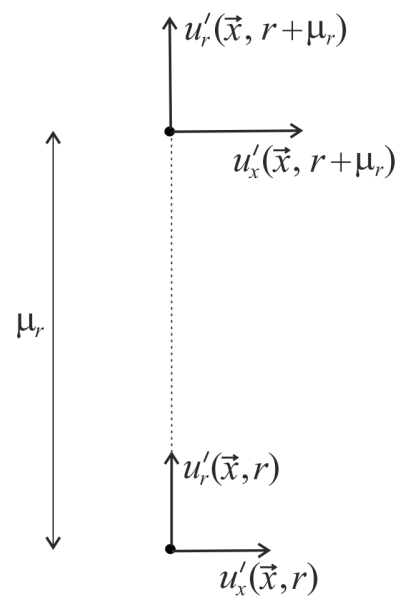

(b)

Fig. 3 Longitudinal and lateral turbulent velocity correlations, with separation vector $\mathbf{r}$ given by: (a) $\mathbf{r}=$ $\left(\vec{\mu}_{x}, 0,0\right) ;(\mathbf{b}) \mathbf{r}=\left(0, \mu_{r}, 0\right)$. 
Batchelor shows that the solution of Eq. 28] is given by

$$
g(r)=f(r)+\frac{1}{2} r \frac{\mathrm{d} f}{\mathrm{~d} r} .
$$

An exact solution of Eq. 29], on assuming that the longitudinal cross-correlation function is a Gaussian function, is given by

$$
\begin{aligned}
& f(r)=\exp \left\{-\frac{\pi}{4}\left(\frac{r^{2}}{\mathcal{L}^{2}}\right)\right\} \\
& g(r)=\left[1-\frac{\pi}{4} \frac{r^{2}}{\mathcal{L}^{2}}\right] \exp \left\{-\frac{\pi}{4}\left(\frac{r^{2}}{\mathcal{L}^{2}}\right)\right\}
\end{aligned}
$$

In three-dimensional homogeneous isotropic turbulence, consider axial, radial and azimuthal separations. For each separation vector $\mathbf{r}$, a pair of longitudinal and lateral velocity cross-correlation functions, $f(r)$ and $g(r)$, are determined by solving Eqs. 3031). For example, for axial separation $\mathbf{r}=\left(\vec{\mu}_{x}, 0,0\right)$,

$$
\begin{aligned}
& \mathcal{R}_{x x}\left(\vec{\mu}_{x}, 0,0\right)=\exp \left\{-\frac{\pi}{4}\left(\frac{\vec{\mu}_{x}^{2}}{\mathcal{L}^{2}}\right)\right\}, \\
& \mathcal{R}_{r r}\left(\vec{\mu}_{x}, 0,0\right)=\left[1-\frac{\pi}{4} \frac{\vec{\mu}_{x}^{2}}{\mathcal{L}^{2}}\right] \exp \left\{-\frac{\pi}{4}\left(\frac{\vec{\mu}_{x}^{2}}{\mathcal{L}^{2}}\right)\right\} .
\end{aligned}
$$

Similar results are found for radial separation $\mathbf{r}=\left(0, \mu_{r}, 0\right)$ and azimuthal separation $\mathbf{r}=(0,0, s)$. Then combining the results for each pair of longitudinal and lateral cross-correlation functions,

$$
\begin{aligned}
& \mathcal{R}_{x x}=\left[1-\frac{\pi}{4} \frac{\mu_{r}^{2}}{\mathcal{L}^{2}}\right]\left[1-\frac{\pi}{4} \frac{s^{2}}{\mathcal{L}^{2}}\right] \exp \left\{-\frac{\pi}{4}\left(\frac{\vec{\mu}_{x}^{2}+\mu_{r}^{2}+s^{2}}{\mathcal{L}^{2}}\right)\right\}, \\
& \mathcal{R}_{r r}=\left[1-\frac{\pi}{4} \frac{\vec{\mu}_{x}^{2}}{\mathcal{L}^{2}}\right]\left[1-\frac{\pi}{4} \frac{s^{2}}{\mathcal{L}^{2}}\right] \exp \left\{-\frac{\pi}{4}\left(\frac{\vec{\mu}_{x}^{2}+\mu_{r}^{2}+s^{2}}{\mathcal{L}^{2}}\right)\right\}, \\
& \mathcal{R}_{\phi \phi}=\left[1-\frac{\pi}{4} \frac{\vec{\mu}_{x}^{2}}{\mathcal{L}^{2}}\right]\left[1-\frac{\pi}{4} \frac{\mu_{r}^{2}}{\mathcal{L}^{2}}\right] \exp \left\{-\frac{\pi}{4}\left(\frac{\vec{\mu}_{x}^{2}+\mu_{r}^{2}+s^{2}}{\mathcal{L}^{2}}\right)\right\} .
\end{aligned}
$$

Cross-correlation functions in the form of Eqs. [34 36] were first published in Powles et al. [6] and McAlpine et al. [7]. In Refs. [6, 7] the turbulent velocity cross-correlation function, formulated based on this type of analysis, is referred to as Gaussian-HIT, i.e. Gaussian $\underline{H}$ omogeneous Isotropic Turbulence. 


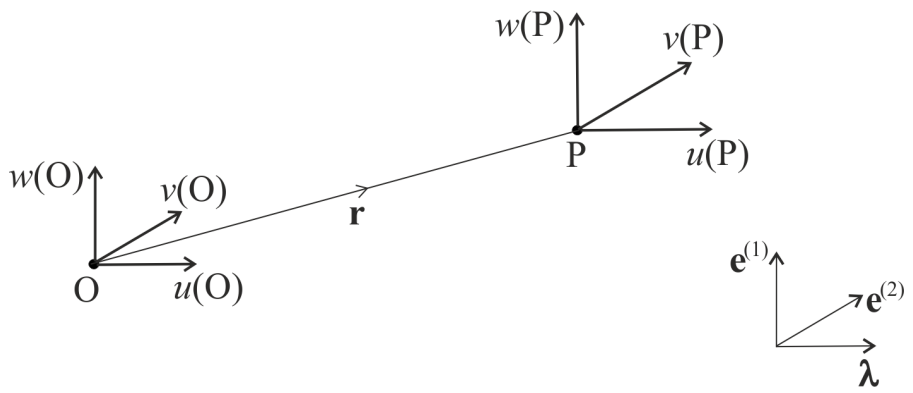

Fig. 4 Orthogonal coordinate system used for Lindborg's analysis of homogeneous axisymmetric turbulence [27].

\section{Homogeneous Axisymmetric Turbulence}

\section{Lindborg's theory}

The theory of homogeneous axisymmetric turbulence was first developed by Batchelor [25] and also Chandrasekhar [26]. However, both representations of $R_{i j}$ for homogeneous axisymmetric turbulence are very complicated. The general form of $R_{i j}$ developed by Batchelor and Chandrasekhar, expressed via a number of unknown scalar functions, linked via complicated relationships, is not amenable to provide simple expressions for cross-correlation functions for homogeneous axisymmetric turbulence.

More recently, Lindborg [27] developed a simpler representation of homogeneous axisymmetric turbulence. In this present work, Lindborg's formulation for homogeneous axisymmetric turbulence has been applied to a circular jet to derive a specific two-point velocity cross-correlation for an axisymmetric turbulent shear layer.

The axis of symmetry is defined by the vector $\lambda$. Lindborg refers to "strong axisymmetry" if there is reflectional symmetry in both planes normal to $\lambda$ and planes containing $\lambda$.

Lindborg defined the orthogonal coordinate system $\left\{\lambda, \mathbf{e}^{(2)}, \mathbf{e}^{(1)}\right\}$ with the unit vectors

$$
\mathbf{e}^{(1)}=\frac{\lambda \times \mathbf{r}}{\rho} \quad \text { and } \quad \mathbf{e}^{(2)}=\mathbf{e}^{(1)} \times \lambda,
$$

where $\rho=|\mathbf{r} \times \lambda|$ and the separation vector $\mathbf{r}$ is the vector from point $\mathrm{O}$ to point $\mathrm{P}$ as shown in Fig.4

Lindborg showed that employing standard Cartesian tensor notation, the two-point velocity correlation tensor for 
homogeneous axisymmetric turbulence can be expressed, invoking various symmetry arguments, as

$$
\begin{aligned}
\widehat{R}_{i j}(\mathbf{r})= & \lambda_{i} \lambda_{j} R_{1}+e_{i}^{(2)} e_{j}^{(2)} R_{2}+e_{i}^{(1)} e_{j}^{(1)} R_{3}+\left(\lambda_{i} e_{j}^{(2)}+\lambda_{j} e_{i}^{(2)}\right) R_{4} \\
& +\left(\lambda_{i} e_{j}^{(1)}+\lambda_{j} e_{i}^{(1)}\right) S_{1}+\left(e_{i}^{(2)} e_{j}^{(1)}+e_{j}^{(2)} e_{i}^{(1)}\right) S_{2},
\end{aligned}
$$

where $R_{1}, R_{2}, R_{3}, R_{4}, S_{1}$ and $S_{2}$ are scalar functions of $\rho$ and $\xi=\mathbf{r} \cdot \lambda$.

These scalar functions are themselves individual two-point velocity correlation functions, based on the velocity components $u, v$ and $w$ in directions $\lambda, \mathbf{e}^{(2)}$ and $\mathbf{e}^{(1)}$ respectively. Then from Lindborg,

$$
\begin{gathered}
R_{1}=\langle u(\mathrm{O}) u(\mathrm{P})\rangle, R_{2}=\langle v(\mathrm{O}) v(\mathrm{P})\rangle, R_{3}=\langle w(\mathrm{O}) w(\mathrm{P})\rangle, R_{4}=\langle u(\mathrm{O}) v(\mathrm{P})\rangle, \\
S_{1}=\langle u(\mathrm{O}) w(\mathrm{P})\rangle \text { and } S_{2}=\langle v(\mathrm{O}) w(\mathrm{P})\rangle .
\end{gathered}
$$

The symmetry arguments mean that

$$
\begin{gathered}
R_{1}(\rho, \xi)=R_{1}(\rho,-\xi), R_{2}(\rho, \xi)=R_{1}(\rho,-\xi), R_{3}(\rho, \xi)=R_{3}(\rho,-\xi), R_{4}(\rho, \xi)=-R_{4}(\rho,-\xi), \\
S_{1}(\rho, \xi)=-S_{1}(\rho,-\xi) \text { and } S_{2}(\rho, \xi)=S_{2}(\rho,-\xi) .
\end{gathered}
$$

Additionally, $R_{1}, R_{2}, R_{3}$ and $S_{2}$ are "even" in $\rho$, whilst $R_{4}$ and $S_{1}$ are "odd" in $\rho$, such that their Taylor series expansions contain only even or odd powers of $\rho$ respectively.

Lindborg applied the continuity condition (27) to Eq. 38 , and obtained

$$
\begin{aligned}
\frac{\partial}{\partial \rho}\left(\rho R_{4}\right) & =-\rho \frac{\partial R_{1}}{\partial \xi} \\
R_{3} & =\frac{\partial}{\partial \rho}\left(\rho R_{2}\right)+\rho \frac{\partial R_{4}}{\partial \xi} \\
\frac{\partial}{\partial \rho}\left(\rho^{2} S_{2}\right) & =-\rho^{2} \frac{\partial S_{1}}{\partial \xi} .
\end{aligned}
$$

Lindborg also found that if there is reflectional symmetry in a plane containing $\lambda$, then the correlation functions $S_{1}$ and $S_{2}$ are zero.

Equations 41,43 are the key theoretical result from Lindborg's paper. These equations are now applied to develop a new two-point turbulent velocity cross-correlation function for an axisymmetric turbulent shear layer. 


\section{Application of Lindborg's theory}

Consider an axisymmetric turbulent shear layer, with parallel and axisymmetric mean flow centred on the $x$-axis, and directed in the positive $x$-direction. Accordingly $\lambda=\mathbf{i}$. Following the procedure utilized for homogeneous isotropic turbulence, the logical approach would be to examine axial, radial and azimuthal separations separately. However, on setting the axial separation vector $\mathbf{r}=\vec{\mu}_{x} \mathbf{i}$, then $\rho=|\mathbf{r} \times \lambda|=0$, and the orthogonal coordinate system $\left\{\lambda, \mathbf{e}^{(2)}, \mathbf{e}^{(1)}\right\}$ is undefined. Furthermore, taking a non-zero azimuthal separation means that the orientation of the orthogonal coordinate system will not be the same at points $\mathrm{O}$ and $\mathrm{P}$. In order to alleviate these problems, a separation vector $\mathbf{r}$ combining both axial and radial but not azimuthal separation is specified. Taking point $\mathrm{O}=\left(\vec{x}_{1}, r_{1}, \phi\right)$ and point $\mathrm{P}=\left(\vec{x}_{2}, r_{2}, \phi\right)$, this gives

$$
\begin{aligned}
\mathbf{r} & =\left(\vec{x}_{1}-\vec{x}_{2}\right) \mathbf{i}+\left(r_{1}-r_{2}\right) \cos \phi \mathbf{j}+\left(r_{1}-r_{2}\right) \sin \phi \mathbf{k}, \\
& =\vec{\mu}_{x} \mathbf{i}+\mu_{r} \cos \phi \mathbf{j}+\mu_{r} \sin \phi \mathbf{k} .
\end{aligned}
$$

In this case, on taking $\lambda=\mathbf{i}$, then

$$
\rho=|\mathbf{r} \times \lambda|=\mu_{r} \quad \text { and } \quad \xi=\mathbf{r} \cdot \lambda=\vec{\mu}_{x},
$$

and from Eq. 37.

$$
\begin{aligned}
& \mathbf{e}^{(1)}=-\sin \phi \mathbf{j}+\cos \phi \mathbf{k}, \\
& \mathbf{e}^{(2)}=\cos \phi \mathbf{j}+\sin \phi \mathbf{k} .
\end{aligned}
$$

Since $\mu_{r}>0$, the unit vectors $\left\{\lambda, \mathbf{e}^{(2)}, \mathbf{e}^{(1)}\right\}$ are equivalent to the unit vectors $\{\hat{\mathbf{x}}, \hat{\mathbf{r}}, \hat{\boldsymbol{\phi}}\}$ associated with the cylindrical polar coordinate system. Also, the separation vector is equivalent to

$$
\mathbf{r}=\vec{\mu}_{x} \hat{\mathbf{x}}+\mu_{r} \hat{\mathbf{r}}
$$

Accordingly, the velocity components $(u, v, w)$ are equivalent to $\left(u_{x}, u_{r}, u_{\phi}\right)$, and the two-point velocity correlation 
functions $R_{1} \rightarrow R_{4}$ can be interpreted from Eq. (39]) as

$$
\begin{aligned}
& R_{1}=\left\langle u_{x}(\mathrm{O}) u_{x}(\mathrm{P})\right\rangle=\mathcal{R}_{x x}(\rho, \xi), \\
& R_{2}=\left\langle u_{r}(\mathrm{O}) u_{r}(\mathrm{P})\right\rangle=\mathcal{R}_{r r}(\rho, \xi), \\
& R_{3}=\left\langle u_{\phi}(\mathrm{O}) u_{\phi}(\mathrm{P})\right\rangle=\mathcal{R}_{\phi \phi}(\rho, \xi), \\
& R_{4}=\left\langle u_{x}(\mathrm{O}) u_{r}(\mathrm{P})\right\rangle=\mathcal{R}_{x r}(\rho, \xi) .
\end{aligned}
$$

These correlation functions are linked via

$$
\begin{aligned}
\frac{\partial}{\partial \rho}\left(\rho \mathcal{R}_{x r}\right) & =-\rho \frac{\partial \mathcal{R}_{x x}}{\partial \xi} \\
\mathcal{R}_{\phi \phi} & =\frac{\partial}{\partial \rho}\left(\rho \mathcal{R}_{r r}\right)+\rho \frac{\partial \mathcal{R}_{x r}}{\partial \xi}
\end{aligned}
$$

from Eqs. 41|42, where $\rho \equiv \mu_{r}$ and $\xi \equiv \vec{\mu}_{x}$.

Owing to the reflectional symmetry in planes containing $\lambda$, the correlation functions $S_{1}$ and $S_{2}$ are zero. This means that the non-isotropic two-point velocity correlation functions $\mathcal{R}_{x \phi}$ and $\mathcal{R}_{r \phi}$ are zero.

An exact solution to Eqs. 50 51] can be derived in terms of the product of a standard Gaussian function 21, and functions of $\vec{\mu}_{x}$ and $\mu_{r}$ which when expanded in terms of Taylor series will contain either even or odd powers of the spatial separation variables. It can be shown that the following expressions 52,55 , provide an exact solution to Eqs. 50 51]:

$$
\begin{aligned}
& \mathcal{R}_{x x}=\left[1-\frac{\pi}{4} \frac{\mu_{r}^{2}}{\mathcal{L}^{2}}\right] \exp \left\{-\frac{\pi}{4}\left(\frac{\vec{\mu}_{x}^{2}+\mu_{r}^{2}+s^{2}}{\mathcal{L}^{2}}\right)\right\}, \\
& \mathcal{R}_{r r}=\left[1-\frac{\pi}{2} \frac{\vec{\mu}_{x}^{2}}{\mathcal{L}^{2}}\right] \exp \left\{-\frac{\pi}{4}\left(\frac{\vec{\mu}_{x}^{2}+\mu_{r}^{2}+s^{2}}{\mathcal{L}^{2}}\right)\right\}, \\
& \mathcal{R}_{\phi \phi}=\left[1-\frac{\pi}{2} \frac{\vec{\mu}_{x}^{2}}{\mathcal{L}^{2}}\right]\left[1-\frac{\pi}{4} \frac{\mu_{r}^{2}}{\mathcal{L}^{2}}\right] \exp \left\{-\frac{\pi}{4}\left(\frac{\vec{\mu}_{x}^{2}+\mu_{r}^{2}+s^{2}}{\mathcal{L}^{2}}\right)\right\}, \\
& \mathcal{R}_{x r}=\frac{\pi}{4 \mathcal{L}^{2}} \vec{\mu}_{x} \mu_{r} \exp \left\{-\frac{\pi}{4}\left(\frac{\vec{\mu}_{x}^{2}+\mu_{r}^{2}+s^{2}}{\mathcal{L}^{2}}\right)\right\} .
\end{aligned}
$$

Note that for homogeneous turbulence, $\mathcal{R}_{x r}(\mathbf{r})=\mathcal{R}_{r x}(-\mathbf{r})$, and from Eq. 55 it follows immediately that $\mathcal{R}_{x r}=\mathcal{R}_{r x}$.

Equations $52-55$ are the key results in this article. The turbulent velocity cross-correlation function formulated based on the theory for homogeneous axisymmetric turbulence is referred to as Gaussian-HAT, i.e. Gaussian 


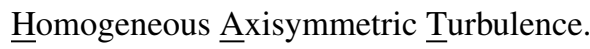

\section{E. Turbulent velocity cross-spectrum}

Combining Eqs. (18) and (22), then substituting into Eq. (11), gives

$$
\begin{gathered}
\Phi_{i j}\left(\bar{r}, K-k, \Gamma_{M}-\gamma_{m}, M-m, \Omega-\omega\right)=\left\langle u_{t i}^{\prime} u_{t j}^{\prime}\right\rangle(\bar{r}) \int_{\vec{\mu}_{x}=-\infty}^{\infty} \int_{\mu_{r}=-\infty}^{\infty} \int_{\mu_{\phi}=-\pi}^{\pi} \int_{\tau=-\infty}^{\infty} \\
\mathcal{F}(\tau) \mathcal{R}_{i j}\left(\vec{\mu}_{x}, \mu_{r}, s\right) e^{\mathrm{i}(K-k) \vec{\mu}_{x}} e^{\mathrm{i}\left(\Gamma_{M}-\gamma_{m}\right) \mu_{r}} e^{\mathrm{i}(M-m) \mu_{\phi}} e^{-\mathrm{i}(\Omega-\omega) \tau} \mathrm{d} \tau \mathrm{d} \mu_{\phi} \mathrm{d} \mu_{r} \mathrm{~d} \vec{\mu}_{x},
\end{gathered}
$$

For convenience, introduce the normalised cross-spectrum function $\widehat{\Phi}_{i j}$ such that

$$
\Phi_{i j}=\left\langle u_{t i}^{\prime} u_{t j}^{\prime}\right\rangle(\bar{r}) \widehat{\Phi}_{i j}
$$

The $\vec{\mu}_{x}$ - and $\mu_{r}$-integrals in Eq. 56 can be evaluated using standard integrals of Gaussian-related functions. The exception is the $\mu_{\phi}$-integral. For all the cross-correlation functions, the $\mu_{\phi}$-integral is the same,

$$
\begin{aligned}
\int_{\mu_{\phi}=-\pi}^{\pi} \exp \left\{-\frac{\pi}{4}\left(\frac{s^{2}}{\mathcal{L}^{2}}\right)\right\} \exp \left\{\mathrm{i}(M-m) \mu_{\phi}\right\} \mathrm{d} \mu_{\phi} & =\int_{\mu_{\phi}=-\pi}^{\pi} \exp \left\{-\frac{\pi}{4}\left(\frac{\bar{r}^{2}}{\mathcal{L}^{2}} \mu_{\phi}^{2}\right)\right\} \exp \left\{\mathrm{i}(M-m) \mu_{\phi}\right\} \mathrm{d} \mu_{\phi}, \\
& =\frac{2 \mathcal{L}}{\bar{r}} \exp \left\{-\frac{(M-m)^{2} \mathcal{L}^{2}}{\pi \bar{r}^{2}}\right\} \operatorname{Re}\left\{\operatorname{erf}\left(\frac{\pi^{3 / 2} \bar{r}}{2 \mathcal{L}}+\mathrm{i} \frac{(M-m) \mathcal{L}}{\pi^{1 / 2} \bar{r}}\right)\right\} .
\end{aligned}
$$

The result from the $\mu_{\phi}$-integral is written in terms of the complex error function erf ( $z$ ) (Appendix, Eq. (91)). The term

$$
\Lambda=\operatorname{Re}\left\{\operatorname{erf}\left(\frac{\pi^{3 / 2} \bar{r}}{2 \mathcal{L}}+\mathrm{i} \frac{(M-m) \mathcal{L}}{\pi^{1 / 2} \bar{r}}\right)\right\}
$$

can be computed, or to avoid this, setting $\Lambda \approx 1$ will be a reasonable approximation for most values of $(M-m)$, provided $\bar{r}>>\mathcal{L}$. (The real part of the complex error function $\operatorname{erf}(z)$ asymptotes to unity if $\operatorname{Re}\{z\}>\operatorname{Im}\{z\}$.) $\operatorname{In}$ fact, setting $\Lambda=1$ leads to the same result that would be obtained if the limits in the $\mu_{\phi}$-integral were $\pm \infty$.

Furthermore, for convenience the function $\Pi_{\tau}$ is defined by

$$
\Pi_{\tau}=\int_{-\infty}^{\infty} \mathcal{F}(\tau) \exp \left\{\mathrm{i}\left([K-k] U_{c}-[\Omega-\omega]\right) \tau\right\} \mathrm{d} \tau
$$


For frozen turbulence, $\mathcal{F}(\tau)=1$, and

$$
\Pi_{\tau}=2 \pi \delta(\zeta)
$$

where

$$
\zeta=[K-k] U_{c}-[\Omega-\omega] .
$$

In the case of non-frozen turbulence, $\mathcal{F}(\tau)$ given by Eq. 23 gives

$$
\Pi_{\tau}=\frac{2(\beta / \mathcal{T})}{(\beta / \mathcal{T})^{2}+\zeta^{2}}
$$

The cross-spectrum functions obtained from the different turbulent velocity cross-correlation functions are summarized below.

\section{Gaussian}

$$
\hat{\Phi}_{i i}=\Pi_{\tau} \Lambda \frac{8 \mathcal{L}^{3}}{\bar{r}} \exp \left\{-\frac{1}{\pi}\left([K-k]^{2} \mathcal{L}^{2}+\left[\Gamma_{M}-\gamma_{m}\right]^{2} \mathcal{L}^{2}+[M-m]^{2} \frac{\mathcal{L}^{2}}{\bar{r}^{2}}\right)\right\}, i i=x x, r r \text { or } \phi \phi .
$$




\section{Gaussian-HIT}

$$
\begin{aligned}
\hat{\Phi}_{x x}= & \Pi_{\tau} \frac{2 \mathcal{L}^{3}}{\bar{r}}\left(1+\frac{2}{\pi}\left[\Gamma_{M}-\gamma_{m}\right]^{2} \mathcal{L}^{2}\right)\left[\left(1+\frac{2}{\pi}[M-m]^{2} \frac{\mathcal{L}^{2}}{\bar{r}^{2}}\right) \Lambda+\frac{\pi \bar{r}}{\mathcal{L}} \cos (M-m) \pi \exp \left\{\frac{(M-m)^{2} \mathcal{L}^{2}}{\pi \bar{r}^{2}}-\frac{\pi^{2} \bar{r}^{2}}{4 \mathcal{L}^{2}}\right\}\right] \times \\
& \exp \left\{-\frac{1}{\pi}\left([K-k]^{2} \mathcal{L}^{2}+\left[\Gamma_{M}-\gamma_{m}\right]^{2} \mathcal{L}^{2}+[M-m]^{2} \frac{\mathcal{L}^{2}}{\bar{r}^{2}}\right)\right\}, \\
\approx & \Pi_{\tau} \Lambda \frac{2 \mathcal{L}^{3}}{\bar{r}}\left(1+\frac{2}{\pi}\left[\Gamma_{M}-\gamma_{m}\right]^{2} \mathcal{L}^{2}\right)\left(1+\frac{2}{\pi}[M-m]^{2} \frac{\mathcal{L}^{2}}{\bar{r}^{2}}\right) \times \\
& \exp \left\{-\frac{1}{\pi}\left([K-k]^{2} \mathcal{L}^{2}+\left[\Gamma_{M}-\gamma_{m}\right]^{2} \mathcal{L}^{2}+[M-m]^{2} \frac{\mathcal{L}^{2}}{\bar{r}^{2}}\right)\right\}, \\
\hat{\Phi}_{r r}= & \Pi_{\tau} \frac{2 \mathcal{L}^{3}}{\bar{r}}\left(1+\frac{2}{\pi}[K-k]^{2} \mathcal{L}^{2}\right)\left[\left(1+\frac{2}{\pi}[M-m]^{2} \frac{\mathcal{L}^{2}}{\bar{r}^{2}}\right) \Lambda+\frac{\pi \bar{r}}{\mathcal{L}} \cos (M-m) \pi \exp \left\{\frac{(M-m)^{2} \mathcal{L}^{2}}{\pi \bar{r}^{2}}-\frac{\pi^{2} \bar{r}^{2}}{4 \mathcal{L}^{2}}\right\}\right] \times \\
& \exp \left\{-\frac{1}{\pi}\left([K-k]^{2} \mathcal{L}^{2}+\left[\Gamma_{M}-\gamma_{m}\right]^{2} \mathcal{L}^{2}+[M-m]^{2} \frac{\mathcal{L}^{2}}{\bar{r}^{2}}\right)\right\}, \\
\approx & \Pi_{\tau} \Lambda \frac{2 \mathcal{L}^{3}}{\bar{r}}\left(1+\frac{2}{\pi}[K-k]^{2} \mathcal{L}^{2}\right)\left(1+\frac{2}{\pi}[M-m]^{2} \frac{\mathcal{L}^{2}}{\bar{r}^{2}}\right) \times \\
& \exp \left\{-\frac{1}{\pi}\left([K-k]^{2} \mathcal{L}^{2}+\left[\Gamma_{M}-\gamma_{m}\right]^{2} \mathcal{L}^{2}+[M-m]^{2} \frac{\mathcal{L}^{2}}{\bar{r}^{2}}\right)\right\}, \\
\hat{\Phi}_{\phi \phi}= & \Pi_{\tau} \Lambda \frac{2 \mathcal{L}^{3}}{\bar{r}}\left(1+\frac{2}{\pi}[K-k]^{2} \mathcal{L}^{2}\right)\left(1+\frac{2}{\pi}\left[\Gamma_{M}-\gamma_{m}\right]^{2} \mathcal{L}^{2}\right) \times \\
& \exp \left\{-\frac{1}{\pi}\left([K-k]^{2} \mathcal{L}^{2}+\left[\Gamma_{M}-\gamma_{m}\right]^{2} \mathcal{L}^{2}+[M-m]^{2} \frac{\mathcal{L}^{2}}{\bar{r}^{2}}\right)\right\} .
\end{aligned}
$$




\section{Gaussian-HAT}

$$
\begin{aligned}
\hat{\Phi}_{x x}= & \Pi_{\tau} \Lambda \frac{4 \mathcal{L}^{3}}{\bar{r}}\left(1+\frac{2}{\pi}\left[\Gamma_{M}-\gamma_{m}\right]^{2} \mathcal{L}^{2}\right) \times \\
& \exp \left\{-\frac{1}{\pi}\left([K-k]^{2} \mathcal{L}^{2}+\left[\Gamma_{M}-\gamma_{m}\right]^{2} \mathcal{L}^{2}+[M-m]^{2} \frac{\mathcal{L}^{2}}{\bar{r}^{2}}\right)\right\}, \\
\hat{\Phi}_{r r}= & \Pi_{\tau} \Lambda \frac{16 \mathcal{L}^{3}}{\pi \bar{r}}[K-k]^{2} \mathcal{L}^{2} \times \\
& \exp \left\{-\frac{1}{\pi}\left([K-k]^{2} \mathcal{L}^{2}+\left[\Gamma_{M}-\gamma_{m}\right]^{2} \mathcal{L}^{2}+[M-m]^{2} \frac{\mathcal{L}^{2}}{\bar{r}^{2}}\right)\right\}, \\
\hat{\Phi}_{\phi \phi}= & \Pi_{\tau} \Lambda \frac{8 \mathcal{L}^{3}}{\pi \bar{r}}[K-k]^{2} \mathcal{L}^{2}\left[1+\frac{2}{\pi}\left[\Gamma_{M}-\gamma_{m}\right]^{2} \mathcal{L}^{2}\right] \times \\
& \exp \left\{-\frac{1}{\pi}\left([K-k]^{2} \mathcal{L}^{2}+\left[\Gamma_{M}-\gamma_{m}\right]^{2} \mathcal{L}^{2}+[M-m]^{2} \frac{\mathcal{L}^{2}}{\bar{r}^{2}}\right)\right\}, \\
\hat{\Phi}_{x r}= & -\Pi_{\tau} \Lambda \frac{8 \mathcal{L}^{3}}{\pi \bar{r}}[K-k] \mathcal{L}\left[\Gamma_{M}-\gamma_{m}\right] \mathcal{L} \times \\
& \exp \left\{-\frac{1}{\pi}\left([K-k]^{2} \mathcal{L}^{2}+\left[\Gamma_{M}-\gamma_{m}\right]^{2} \mathcal{L}^{2}+[M-m]^{2} \frac{\mathcal{L}^{2}}{\bar{r}^{2}}\right)\right\} .
\end{aligned}
$$

\section{Results}

The measurements of haystacking taken in the Von Karman Institute wind tunnel and published by Candel, Guedel and Julienne [1] in 1975 are used for validation purposes to compare against results obtained using the turbulent velocity cross-correlation functions formulated in this article.

The experimental set-up is shown in Fig 5 A monochromatic sound source was positioned on the jet centreline, at two axial stations $x=0.5 \mathrm{~m}$ and $1.95 \mathrm{~m}$, measured downstream of the jet's circular nozzle. A microphone was located outside the jet. The source's directivity was fairly uniform, especially around polar angle equal to $90^{\circ}$ where the measurements were acquired.

In Ref. [1] it is shown that the shear layer velocity profile $U(r)$ is given approximately by

$$
\frac{U}{U_{J}}=\frac{1}{2}(1-\tanh \eta)
$$

where

$$
\eta=\frac{2}{\delta}\left(r-r_{c}\right)
$$




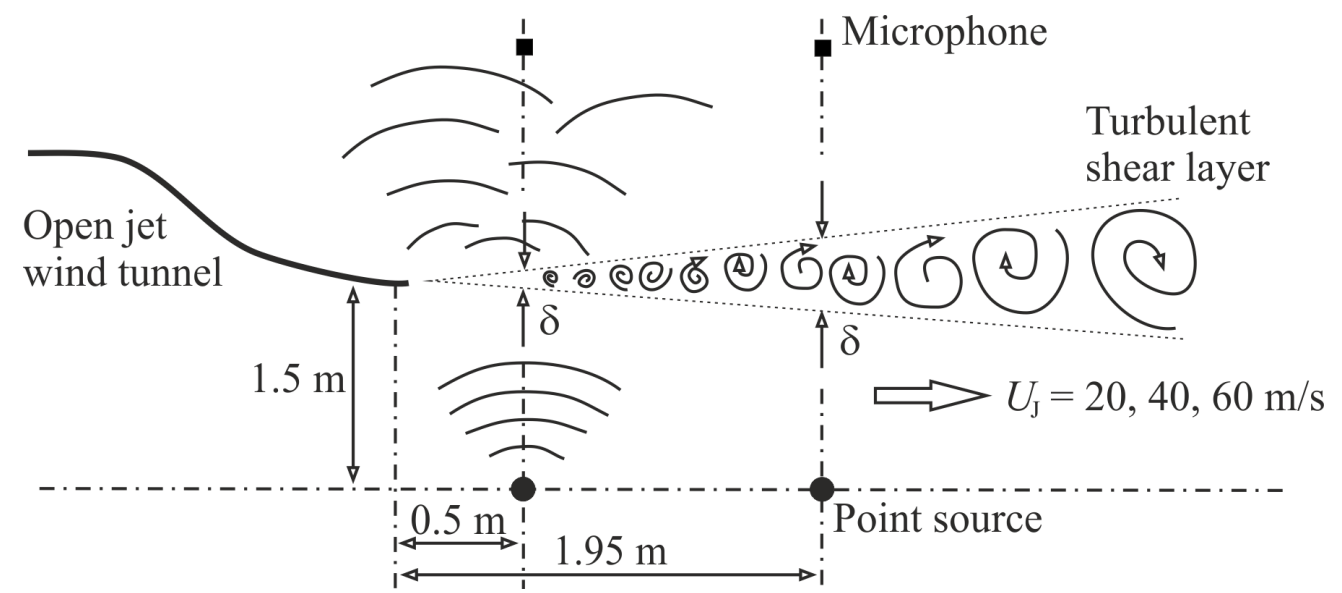

Fig. 5 Sketch of experimental set-up used by Candel et al. [1]. A point source is placed on the centreline of a jet, and a microphone placed outside the jet at a polar angle approximately $90^{\circ}$ from the source. Taken from Ref. [7]

In the simulations, using the estimates given by Candel et al. [1], the shear layer thickness $\delta=0.17 x$, and the jet radius $r_{c}=1.47+0.048 x$.

In reality, within the turbulent shear layer the components of the Reynolds stress (17) vary with radial position. Measured profiles of $\left\langle u_{x t}^{\prime 2}\right\rangle(\eta)$ and $\left\langle u_{r t}^{\prime 2}\right\rangle(\eta)$ are in Ref. [1], but there are no measurements of $\left\langle u_{\phi_{t}}^{\prime 2}\right\rangle(\eta)$ or $\left\langle u_{x t}^{\prime} u_{r t}^{\prime}\right\rangle(\eta)$. Following Hussein et al. [20], the isotropic terms of the Reynolds stress are modelled by functions of the form $\left(C_{0}+C_{2} \eta^{2}\right) \exp \left\{-A \eta^{2}\right\}$, whereas the non-isotropic term is modelled by the function $\left(C_{1} \eta\right) \exp \left\{-B \eta^{2}\right\}$. The coefficients of the functions for $\left\langle u_{x t}^{\prime 2}\right\rangle(\eta)$ and $\left\langle u_{r t}^{\prime 2}\right\rangle(\eta)$ are determined by fitting the curves to the measured profiles from Ref. [1]. The maximum value of the axial turbulence intensity is about $16 \%$, and the maximum radial turbulence intensity is about $12 \%$. Although measurements of $\left\langle u_{\phi_{t}}^{\prime 2}\right\rangle(\eta)$ and $\left\langle u_{x t}^{\prime} u_{r t}^{\prime}\right\rangle(\eta)$ are not available, the former can be assumed the same as $\left\langle u_{r t}^{\prime 2}\right\rangle(\eta)$ in a circular jet, cf. Townsend [28] (p. 192). The remaining non-isotropic component is estimated utilising the measurements (of a different smaller circular jet) in Ref. [20]. These measurements indicate that the maximum value of $\left\langle u_{x t}^{\prime} u_{r t}^{\prime}\right\rangle$ is approximately $25 \%$ of the maximum value of $\left\langle u_{x t}^{\prime 2}\right\rangle$.

The haystacking measurements from Ref. [1] show the power spectral density normalized by the total power, measured at the reference microphone $\left(\Theta=90^{\circ}\right)$. The levels are plotted against the difference between the scattered and source frequencies, i.e. $F-f$ where $\Omega=2 \pi F$ and $\omega=2 \pi f$. In the relevant examples, reproduced from Ref. [1] in Fig. 6. the velocity of the jet was varied between $U_{J}=20 \mathrm{~ms}^{-1}$ and $60 \mathrm{~ms}^{-1}$, and the source frequency was varied between 4 and $20 \mathrm{kHz}$. Two of the results from Ref. [1] show measurements of how the levels vary with source frequency at axial stations $1(0.5 \mathrm{~m})$ and $2(1.95 \mathrm{~m})$, Fig. 6.a)\&(c), for fixed jet velocity $U_{J}=60 \mathrm{~ms}^{-1}$. The other result 
shows measurements of how the levels vary with jet velocity at axial station 1, Fig. 6. b), for fixed source frequency $f=20 \mathrm{kHz}$.

Since a monochromatic source on the jet centreline is used in the experiments, the incident field is single-frequency and axisymmetric, i.e. azimuthal order $m=0$. In this case, evaluation of the scattered field given by Eq. (10) is considerably simplified because only a single integration over the incident field polar angle $\theta$ is required. (Full details of this simplification are in Ref. [7, §2.7, p. 3818].)

Furthermore, Ref. [1] has some measurements of the incident field, but not for the whole polar arc. Nevertheless, since the haystacking measurements are at $90^{\circ}$, the measured directivity pattern for the $20 \mathrm{kHz}$ tone [1, Fig. 16, p. 292] between 60 and $120^{\circ}$ has been used in all the simulations. As a check, also the simulated results have been calculated with the assumption that the incident field directivity is uniform. In fact since the directivity is fairly uniform around $90^{\circ}$, there is little difference in the simulated results at $90^{\circ}$ using the measured or uniform directivity.

All the models of the turbulence spectrum require parameters to characterize the turbulence. These are the convection velocity of the turbulence $U_{c}$, the turbulence integral lengthscale $\mathcal{L}$, and for non-frozen turbulence, the integral timescale $\mathcal{T}$. Candel et al. [1] propose the following estimates: $U_{c}=0.5 U_{J}$ and $l \approx 3.2 \delta$, where $l$ is an arbitrary lengthscale. There is no estimate in Ref. [1] of a timescale.

The measured haystacks exhibit a "double-humped" structure. The lengthscale was chosen in Ref. [1] such that the peaks in the lateral sidebands of the measured haystacks occur with frequency shift (in Hz) given by

$$
|F-f| \approx \frac{U_{c}}{l}
$$

In Ref. [7 §3.3, pp. 3820-3821], a slightly different expression for the frequency shift was proposed. The expression was derived by determining the turning points of the function (referred to as $g(x)$ ) which characterises the Gaussian-HIT turbulent velocity spectrum. The same approach has been used in this article, except by taking the function which characterises the Gaussian-HAT spectrum, viz.

$$
g(x)=x^{2} \mathrm{e}^{-x^{2} / \pi}
$$

(For an explanation why this is the relevant function, refer to Sec. $\mathrm{V}$ ) Then following the same approach in Ref. [7] gives

$$
|F-f|=\frac{1}{2 \sqrt{\pi}} \frac{U_{c}}{\mathcal{L}}
$$




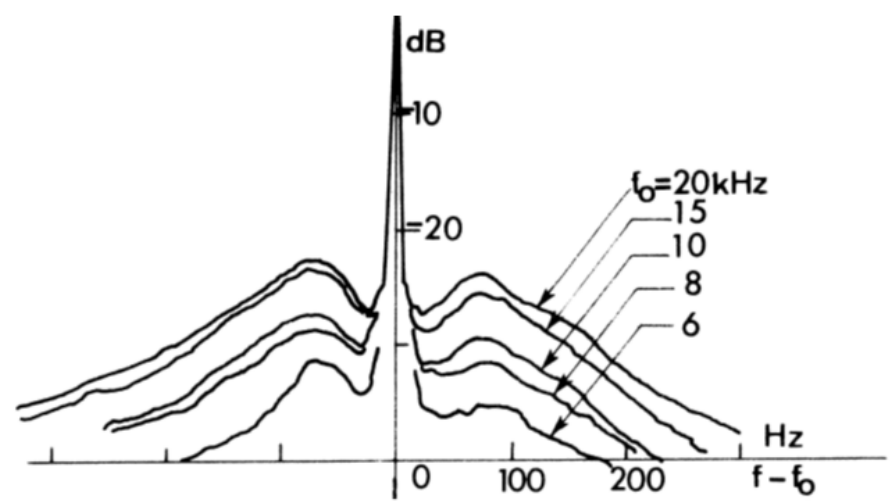

(a) Variation with source frequency at jet velocity $U_{J}=60 \mathrm{~ms}^{-1}$ Thin shear layer.

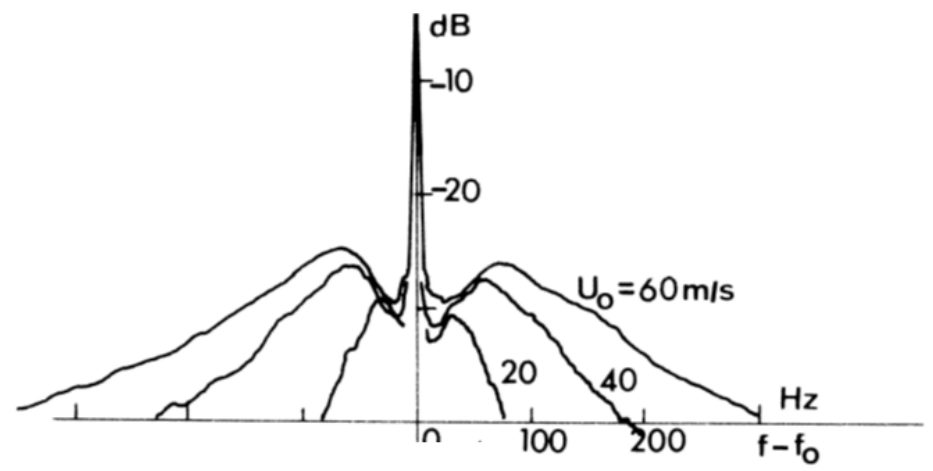

(b) Variation with jet velocity at tone frequency $f=20 \mathrm{kHz}$ Thin shear layer.

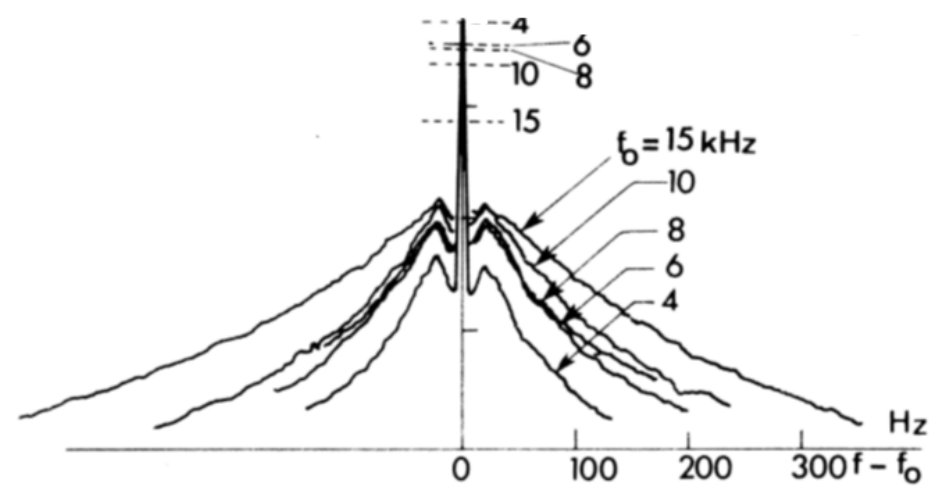

(c) Variation with source frequency at jet velocity $U_{J}=60 \mathrm{~ms}^{-1}$ Thick shear layer.

Fig. 6 Measurements of power spectral density normalized by the total power. (Reproduced from Candel et al. [1, p. 295, Fig. 25(a-c)]. (C1975 IEEE 
Therefore, following Ref. [1], the lengthscale has been chosen such that the frequency shift given by Eq. 76] matches the measured double-humped haystacks. The timescale is only required for the non-frozen turbulence model. Since no estimates for $\mathcal{T}$ are available, its value has been chosen to try to best match the shape of the haystacks' profiles with the measurements.

In summary, in all the simulations, the parameters were

$$
U_{c}=0.5 U_{J}, \quad \mathcal{L}=1.38 \delta, \quad \mathcal{T}=0.7 \frac{\mathcal{L}}{U_{c}}
$$

Figures 7, 8 and 9 show the simulations with the Gaussian, Gaussian-HIT and Gaussian-HAT turbulent velocity cross-correlation models respectively, based on the three examples of measured haystacks in Fig. 6 from Candel et al. [1]. These simulations are all for non-frozen turbulence. These examples show simulations at axial station 1, varying the source frequency for fixed jet velocity $U_{J}=60 \mathrm{~ms}^{-1}$, or varying the jet velocity for fixed source frequency $f=20 \mathrm{kHz}$. Additionally the examples show simulations at axial station 2, where the shear layer is thicker compared to the shear layer at axial station 1, varying the source frequency for fixed jet velocity $U_{J}=60 \mathrm{~ms}^{-1}$. The non-frozen turbulence model leads to simulated haystacks that more closely resemble the shape of the measured haystacks. In order to illustrate this, Fig. 10 show the comparable simulations with the Gaussian-HAT spectrum and frozen turbulence. Comparing this with Fig. 9 it is immediately clear that the non-frozen turbulence model gives haystacks' predictions with concave shape, similar to the measured haystacks in Fig.6 6

All the haystack simulations shown in Figures $7+10$ are calculated by summing the azimuthal orders from $M=-50$ to 50. The tone levels are not directly calculated from Equation (10) since this expression is for the scattered field. Instead the reduced level of the tone owing to the scattering is calculated by assuming conservation of energy, i.e. the energy of the incident tone before the scattering is assumed to equal the resulting energy in the tone and haystack. Furthermore, each of the simulated haystacks has been normalised based on the total power in the spectrum.

\section{Discussion}

The choice of model to describe the turbulence spectrum, and whether the model assumes frozen or non-frozen turbulence, significantly affects the shape of the predicted haystacks. Furthermore, a parametric study in McAlpine, Powles \& Tester [7] (Sec. 3.5, Figures 11-13) showed how varying the convection velocity $U_{c}$, integral lengthscale $\mathcal{L}$ or integral timescale $\mathcal{T}$ affected the shape of the predicted haystacks. The choice of convection velocity and lengthscale can significantly affect both the peak level and width of the haystack. However the choice of timescale does not 


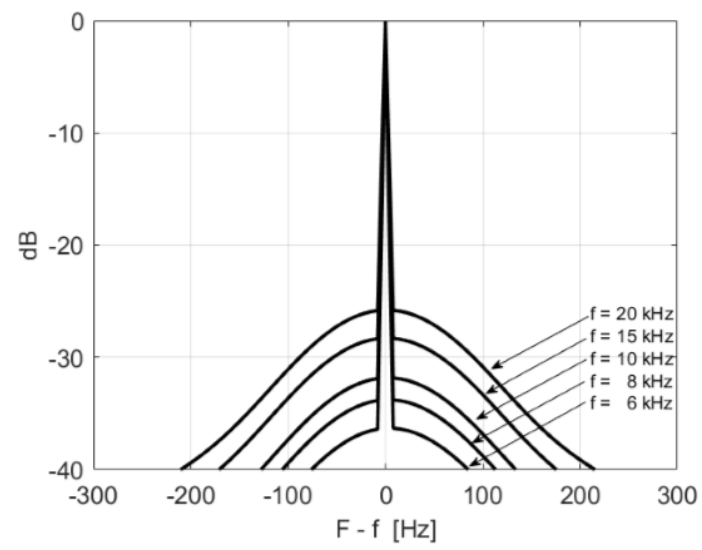

(a) Variation with source frequency at jet velocity $U_{J}=60 \mathrm{~ms}^{-1}$ Thin shear layer.

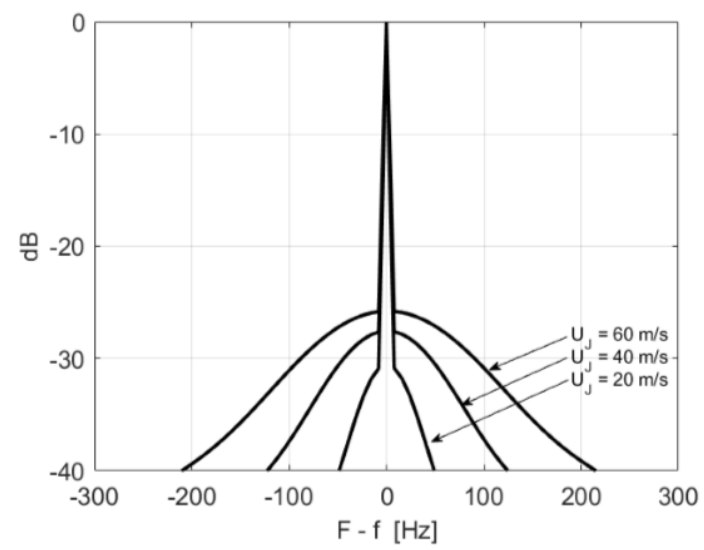

(b) Variation with jet velocity at tone frequency $f=20 \mathrm{kHz}$ Thin shear layer.

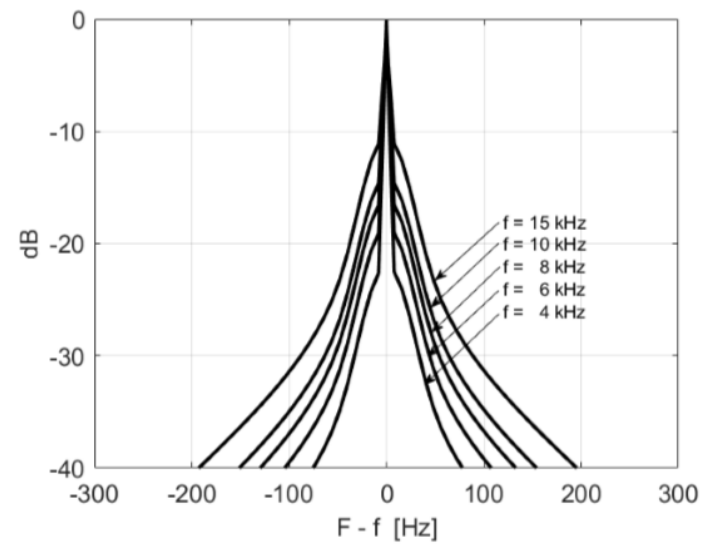

(c) Variation with source frequency at jet velocity $U_{J}=60 \mathrm{~ms}^{-1}$ Thick shear layer.

Fig. 7 Predictions of the normalised far-field power spectral density at $\Theta=90^{\circ}$, based on the haystacking results from Candel et al. [1, p. 295, Fig. 25(a-c)]. Predictions use the Gaussian correlation function with $U_{c} / U_{J}=0.5, \mathcal{L} / \delta=1.38$, and $\mathcal{T} U_{c} / \mathcal{L}=0.7$. 


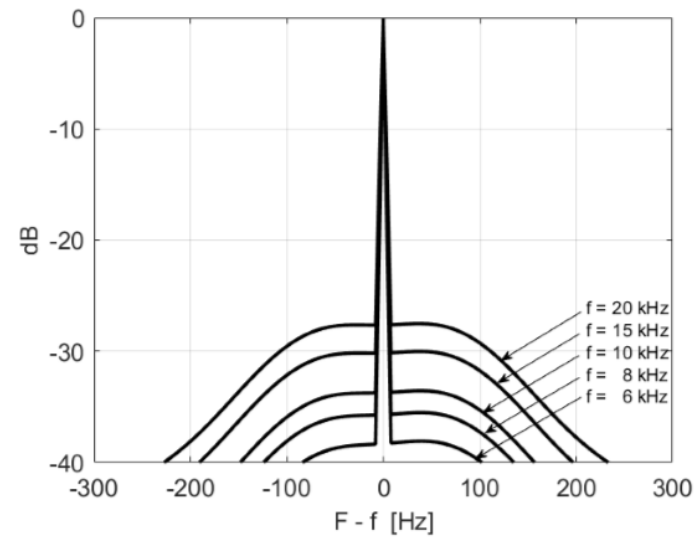

(a) Variation with source frequency at jet velocity $U_{J}=60 \mathrm{~ms}^{-1}$ Thin shear layer.

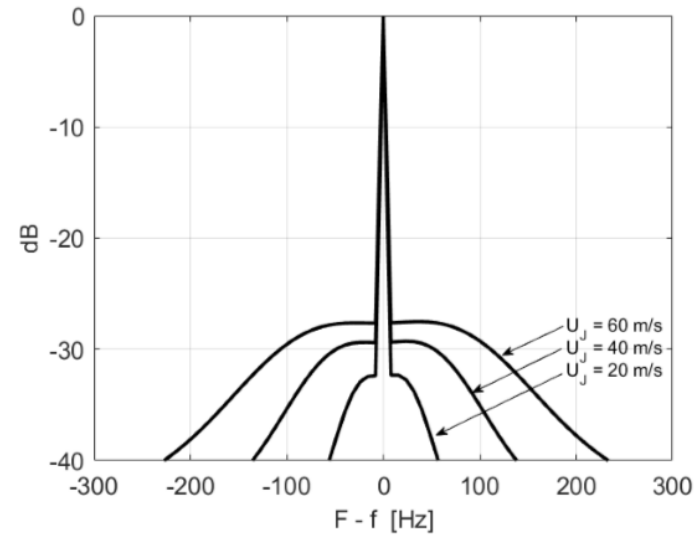

(b) Variation with jet velocity at tone frequency $f=20 \mathrm{kHz}$ Thin shear layer.

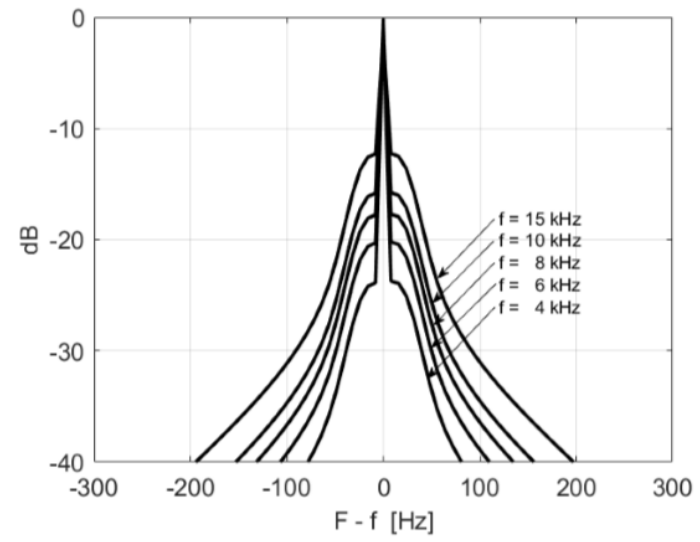

(c) Variation with source frequency at jet velocity $U_{J}=60 \mathrm{~ms}^{-1}$ Thick shear layer.

Fig. 8 Predictions of the normalised far-field power spectral density at $\Theta=90^{\circ}$, based on the haystacking results from Candel et al. [1, p. 295, Fig. 25(a-c)]. Predictions use the Gaussian-HIT correlation function with $U_{c} / U_{J}=0.5, \mathcal{L} / \delta=1.38$, and $\mathcal{T} U_{c} / \mathcal{L}=0.7$. 


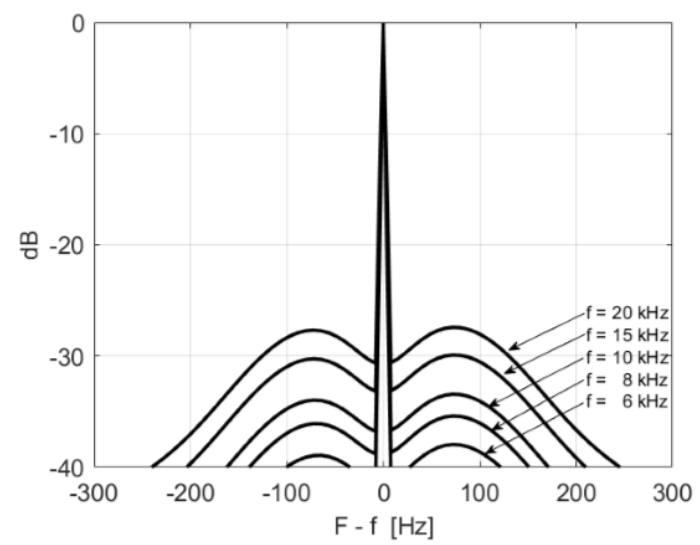

(a) Variation with source frequency at jet velocity $U_{J}=60 \mathrm{~ms}^{-1}$ Thin shear layer.

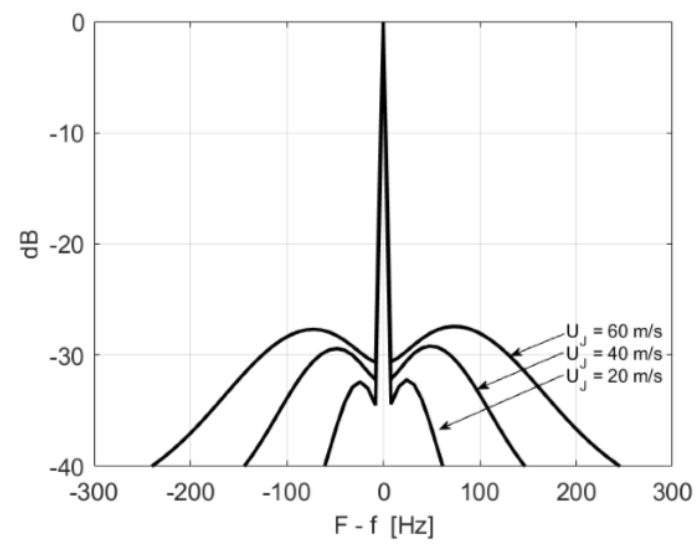

(b) Variation with jet velocity at tone frequency $f=20 \mathrm{kHz}$ Thin shear layer.

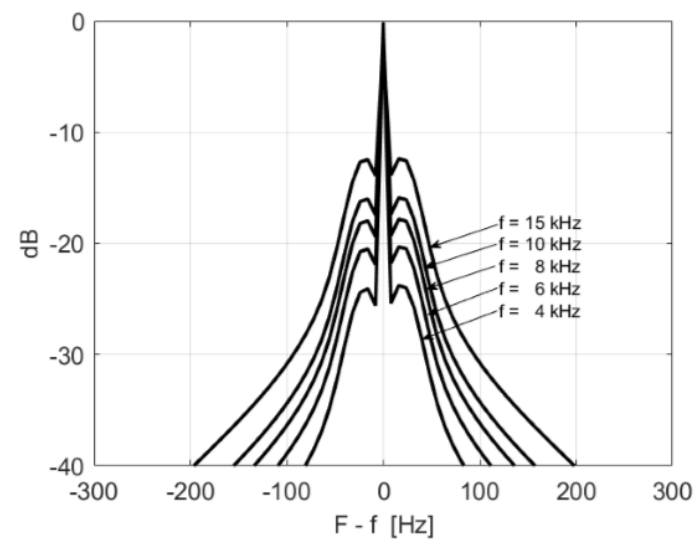

(c) Variation with source frequency at jet velocity $U_{J}=60 \mathrm{~ms}^{-1}$ Thick shear layer.

Fig. 9 Predictions of the normalised far-field power spectral density at $\Theta=90^{\circ}$, based on the haystacking results from Candel et al. [1, p. 295, Fig. 25(a-c)]. Predictions use the Gaussian-HAT correlation function with $U_{c} / U_{J}=0.5, \mathcal{L} / \delta=1.38$, and $\mathcal{T} U_{c} / \mathcal{L}=0.7$. 


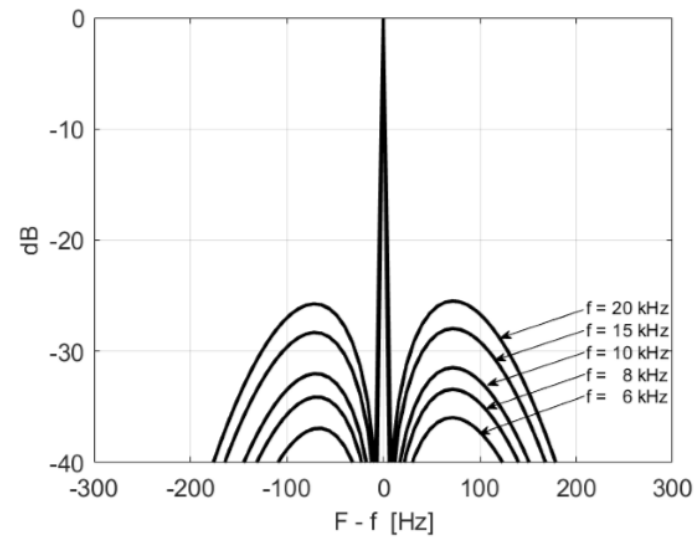

(a) Variation with source frequency at jet velocity $U_{J}=60 \mathrm{~ms}^{-1}$ Thin shear layer.

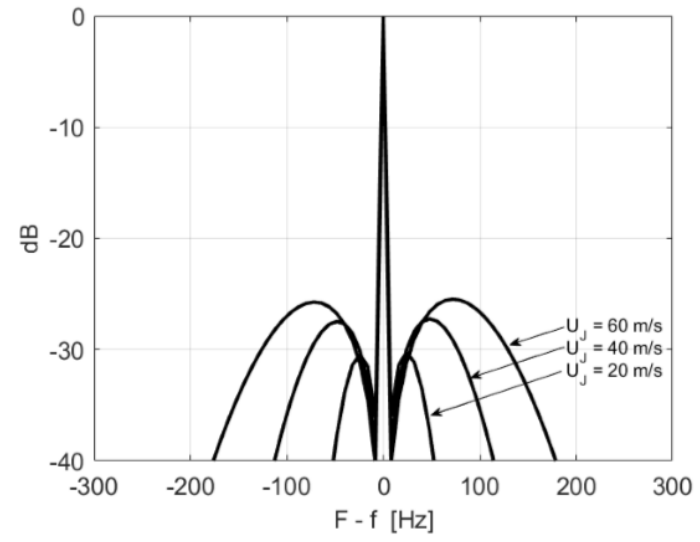

(b) Variation with jet velocity at tone frequency $f=20 \mathrm{kHz}$ Thin shear layer.

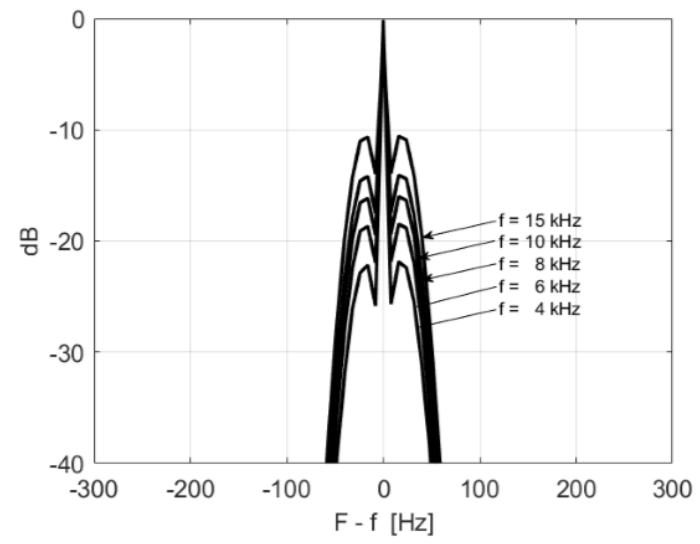

(c) Variation with source frequency at jet velocity $U_{J}=60 \mathrm{~ms}^{-1}$ Thick shear layer.

Fig. 10 Predictions of the normalised far-field power spectral density at $\Theta=90^{\circ}$, based on the haystacking results from Candel et al. [1, p. 295, Fig. 25(a-c)]. Predictions use the Gaussian-HAT correlation function with $U_{c} / U_{J}=0.5, \mathcal{L} / \delta=1.4$, and $\mathcal{T} U_{c} / \mathcal{L}=1.0$, i.e. frozen gărbulence. 
significantly affect the peak level, only the width of the haystack and its predicted shape which is more concave compared to the predicted shape of the haystack with frozen turbulence.

The results in $\S \mathrm{IV}$ were obtained using values of the parameters $U_{c}, \mathcal{L}$ and $\mathcal{T}$ that were selected to provide a close match to the measured data. As explained in the previous section, the ratio of $U_{c} / \mathcal{L}$ is determined via Eq. 766 to match the frequency shift observed in the measured haystacks. The best match between measurement and prediction is observed in the simulations calculated with the Gaussian-HAT turbulent velocity cross-spectrum with non-frozen turbulence - compare Figs. 6 and 9 The double humped haystacks are located, approximately, at the correct frequency shift from the tone. Also the levels of the haystacks, relative to the tone, are roughly comparable. All the examples show weak-scattering; each haystack is at least $10 \mathrm{~dB}$ lower than the tone.

In the absence of detailed measurements of the turbulence, it is not possible to verify whether the values used of $U_{c}, \mathcal{L}$ and $\mathcal{T}$ are realistic. Candel et al. [1] estimated an "eddy scale" $l \approx 3.2 \delta$, based on the large-scale turbulent structures. In the current work, the lengthscale used in the simulations was varied to find the value of $\mathcal{L}$ which gave the best simulation results. Using Eq. (76) the measured peaks in the lateral sidebands of the haystacks are reproduced in the predictions if the lengthscale $\mathcal{L}$ is comparable to the shear layer thickness $\delta$. This supports the assertion in Candel et al. that it is large-scale turbulent structures that control the shape of the haystacks. Specifically it was found that at axial station 1, where the shear layer is relatively thin, more realistic predictions were found with $\mathcal{L}$ greater than $\delta$. Conversely, at axial station 2, which is further downstream where the shear layer is relatively thick, more realistic predictions were found with $\mathcal{L}$ less than $\delta$. Thus, the selected value of $\mathcal{L}=1.38 \delta$ was chosen as a compromise to best match the results with both the thin and thick turbulent shear layer.

Returning to the turbulence spectrum, the terms of the turbulent velocity cross-spectral matrix are grouped within the square brackets in Eq. 16. The simulations with the Gaussian or Gaussian-HIT spectra only include the isotropic terms $\Phi_{x x}, \Phi_{r r}$ and $\Phi_{\phi \phi}$, whereas using the Gaussian-HAT spectra for an axisymmetric turbulent shear layer also includes the non-isotropic term $\Phi_{x r}$.

These terms are

$$
\left[K^{2} \Phi_{x x}+\bar{\Gamma}_{M}^{2} \Phi_{r r}+(M / \bar{r})^{2} \Phi_{\phi \phi}+2 K \bar{\Gamma}_{M} \Phi_{x r}\right]=K^{2} \Phi_{x x}+\left(\frac{D^{2}}{\overline{c^{2}}}-K^{2}-\frac{M^{2}}{\bar{r}^{2}}\right) \Phi_{r r}+\frac{M^{2}}{\bar{r}^{2}} \Phi_{\phi \phi}+2 K \bar{\Gamma}_{M} \Phi_{x r}
$$

The simulations in $\S \mathrm{IV}$ are at $\Theta=90^{\circ}$, therefore $K=\left|K_{\infty}\right| \cos \Theta=0$, and also $D=\Omega$. Hence, Eq. 78 reduces to 

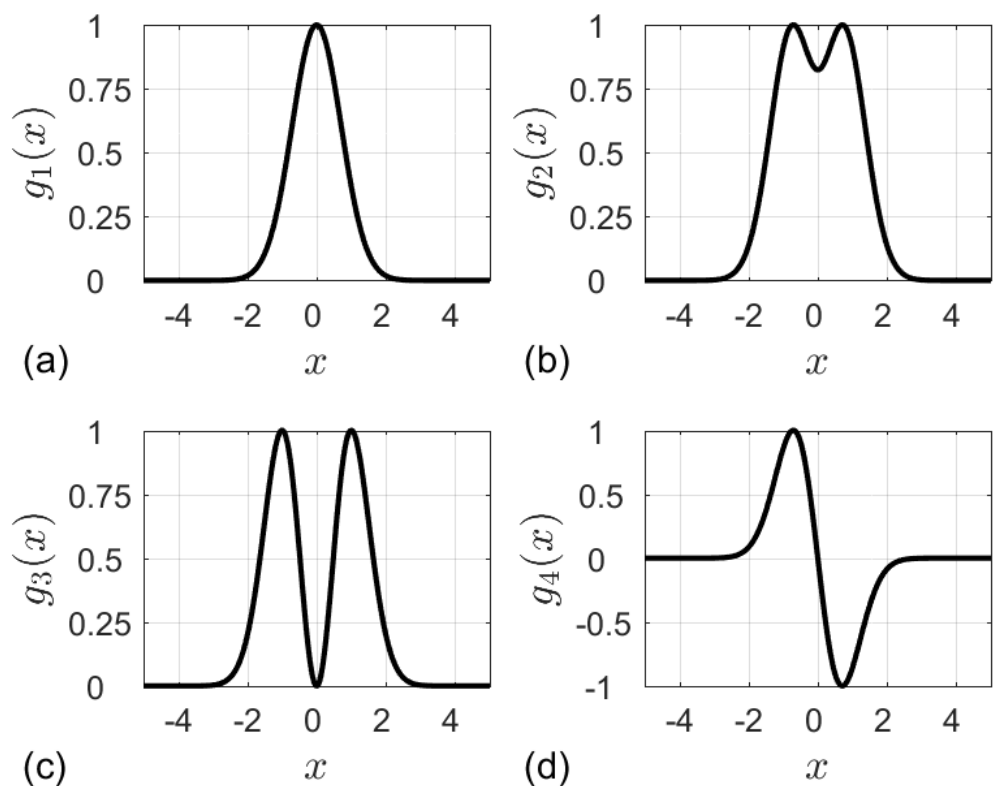

Fig. 11 Gaussian function and modified Gaussian functions: (a) $g_{1}(x)=\exp \left\{-x^{2}\right\}$; (b) $g_{2}(x)=$ $\left[1+2 x^{2}\right] \exp \left\{-x^{2}\right\}$; (c) $g_{3}(x)=x^{2} \exp \left\{-x^{2}\right\}$; (d) $g_{4}(x)=-x \exp \left\{-x^{2}\right\}$. (Functions normalised setting maximum value equal to unity.)

approximately

$$
[\ldots]=\frac{\Omega^{2}}{\overline{c^{2}}} \Phi_{r r}+\frac{M^{2}}{\bar{r}^{2}}\left(\Phi_{\phi \phi}-\Phi_{r r}\right) \approx \frac{\Omega^{2}}{\overline{c^{2}}} \Phi_{r r}
$$

assuming that $\Phi_{r r}$ and $\Phi_{\phi \phi}$ are the same (or similar) magnitude. Thus, the key term which determines the shape of the haystack at $\Theta=90^{\circ}$ is $\Phi_{r r}$, i.e. the radial-radial quadrupole.

Now compare the term $\Phi_{r r}$ for the Gaussian, Gaussian-HIT and Gaussian-HAT spectra, Eqs. [64), (66) and 69) respectively. The shape of the term $\Phi_{r r}$ is characterised by the following functions:

$$
\begin{aligned}
\text { Gaussian } & g_{1}(x)=\exp \left\{-x^{2}\right\}, \\
\text { Gaussian-HIT } & g_{2}(x)=\left[1+2 x^{2}\right] \exp \left\{-x^{2}\right\}, \\
\text { Gaussian-HAT } & g_{3}(x)=x^{2} \exp \left\{-x^{2}\right\} .
\end{aligned}
$$

These functions are shown plotted in Fig. $11(\mathrm{a}-\mathrm{c})$. 
Equations (81] or [82] are the functions that characterize the Gaussian-HIT or Gaussian-HAT correlations respectively. Both functions exhibit a double-humped shape, but the function associated with the Gaussian-HAT correlation has the far more pronounced double-hump. This can be directly connected to the two-dimensional problem for homogeneous isotropic turbulence. The theory in $\S$ III.C is slightly modified if $\mathbf{r}=\left(\vec{\mu}_{x}, \mu_{r}\right)$ is a two-dimensional vector. In this case, Ewert [22] showed that

$$
g(r)=f(r)+r \frac{\mathrm{d} f}{\mathrm{~d} r}
$$

replaces Eq. 29) if $\mathbf{r}$ is two-dimensional.

For this idealised two-dimensional problem, an exact solution, on assuming that the longitudinal cross-correlation function is given by a Gaussian function, is given by

$$
\begin{aligned}
& f(r)=\exp \left\{-\frac{\pi}{4}\left(\frac{r^{2}}{\mathcal{L}^{2}}\right)\right\} \\
& g(r)=\left[1-\frac{\pi}{2} \frac{r^{2}}{\mathcal{L}^{2}}\right] \exp \left\{-\frac{\pi}{4}\left(\frac{r^{2}}{\mathcal{L}^{2}}\right)\right\} .
\end{aligned}
$$

Comparing the functions $f$ and $g$ for the three-dimensional problem (Eqs. 30[31), and the two-dimensional problem (Eqs. 84/85), the only difference is the function $g$ where the factor $\pi / 4$ is replaced by $\pi / 2$ in the term in square brackets. The significance of this seemingly minor difference in the two functions for $g(r)$ is seen on taking the Fourier transforms, i.e.

$$
\begin{aligned}
& \text { 3D } \quad \int_{r=-\infty}^{\infty}\left[1-\frac{\pi}{4} \frac{r^{2}}{\mathcal{L}^{2}}\right] \exp \left\{-\frac{\pi}{4}\left(\frac{r^{2}}{\mathcal{L}^{2}}\right)\right\} \exp \{\mathrm{i} \alpha r\} \mathrm{d} r=\mathcal{L}\left(1+2 \frac{\alpha^{2} \mathcal{L}^{2}}{\pi}\right) \exp \left\{-\frac{\alpha^{2} \mathcal{L}^{2}}{\pi}\right\}, \\
& \text { 2D } \quad \int_{r=-\infty}^{\infty}\left[1-\frac{\pi}{2} \frac{r^{2}}{\mathcal{L}^{2}}\right] \exp \left\{-\frac{\pi}{4}\left(\frac{r^{2}}{\mathcal{L}^{2}}\right)\right\} \exp \{\mathrm{i} \alpha r\} \mathrm{d} r=4 \mathcal{L} \frac{\alpha^{2} \mathcal{L}^{2}}{\pi} \exp \left\{-\frac{\alpha^{2} \mathcal{L}^{2}}{\pi}\right\} \text {. }
\end{aligned}
$$

The shape of the spectrum for the three-dimensional case is in the form given by $g_{2}(x) \sqrt[81]{\text {, }}$, shown in Fig. 11 b). By comparison the spectrum for the two-dimensional case is in the form given by $g_{3}(x)$ [82, shown in Fig. 11)(c). In the latter case, the spectrum exhibits a pronounced double-humped shape. In fact it is the function $g_{3}(x)$ that characterises the term $\Phi_{r r}$ in the Gaussian-HAT spectrum. Therefore the Gaussian-HAT spectrum for the axisymmetric case, and the idealised two-dimensional case, are closely linked. This is as expected since the axisymmetric case is quasi-two-dimensional, with the statistical properties of the flow being dependent on $x$ and $r=\sqrt{y^{2}+z^{2}}$ only. In 
practice the double-humped shape is less pronounced than the two-dimensional case owing to the inclusion of the temporal decay function.

\section{Conclusion}

The Gaussian-HAT cross-correlation and its cross-spectrum, appears to provide a suitable form to be used for simulations of spectral broadening of a tonal sound field propagating through an axisymmetric turbulent shear layer. Using the Gaussian-HAT cross-spectrum, the weak-scattering method is shown to produce realistic simulations of haystacking, but judicious choice of some of the turbulence parameters is required in order to replicate the measurements. The spectral shape of the haystacks is similar to the shape of the turbulent velocity cross-spectrum, including a doublehumped structure centred on the tone. It is necessary to include a temporal decay function with a characteristic time scale, in order to predict haystacks with a concave shape.

Since the statistical properties of the turbulence in an axisymmetric circular jet depend only on $x$ and $r$, the homogeneous axisymmetric turbulence model is directly comparable to a quasi-two-dimensional model based on homogeneous isotropic turbulence. It is shown that the homogeneous axisymmetric turbulence model gives more realistic predictions of haystacks compared to utilising a three-dimensional model based on homogeneous isotropic turbulence.

The new Gaussian-HAT cross-correlation function developed specifically for application to an axisymmetric turbulent shear layer requires further validation with a more comprehensive dataset. Ideally this would include measurements of spectral broadening over a range of polar angles, more detailed aerodynamic measurements of the shear layer, and, importantly, measurements of the sound field within the jet, before the sound has propagated through the turbulent shear layer and undergone spectral broadening.

All non-proprietary data supporting this study are openly available from the University of Southampton repository at https://doi.org/10.5258/SOTON/D1045.

\section{Integral timescale for non-frozen turbulence modelling}

In the case of non-frozen turbulence, on taking a Gaussian or modified Gaussian cross-correlation function, the integral timescale is found by evaluating the integral

$$
\mathcal{T}=\int_{0}^{\infty} \mathcal{F}(\tau) \exp \left\{-\frac{\pi}{4}\left(\frac{\left[-U_{c} \tau\right]^{2}}{\mathcal{L}^{2}}\right)\right\} \mathrm{d} \tau,
$$


from the definition of $\mathcal{T}$ in Eq. 20 .

On substituting for the temporal function $\mathcal{F}(\tau)[23$, the integral timescale $\mathcal{T}$ is given by

$$
\begin{aligned}
\mathcal{T} & =\int_{0}^{\infty} \exp \left\{-\frac{\beta|\tau|}{\mathcal{T}}-\frac{\pi}{4}\left(\frac{\left[-U_{c} \tau\right]^{2}}{\mathcal{L}^{2}}\right)\right\} \mathrm{d} \tau \\
& =\frac{\mathcal{L}}{U_{c}} \exp \left\{\frac{\mathcal{L}^{2} \beta^{2}}{\pi U_{c}^{2} \mathcal{T}^{2}}\right\}\left[1-\operatorname{erf}\left(\frac{\beta \mathcal{L}}{\sqrt{\pi} \mathcal{T} U_{c}}\right)\right],
\end{aligned}
$$

where

$$
\operatorname{erf}(z)=\frac{2}{\sqrt{\pi}} \int_{0}^{z} e^{-t^{2}} \mathrm{~d} t
$$

is the error function.

On setting $\beta=0$, since erf $(0)=0$, Eq. $(90)$ reduces to $\mathcal{T}=\mathcal{L} / U_{c}$, i.e. the timescale for frozen turbulence.

For $\beta>0$, Eq. 90, can be written more succinctly as

$$
\epsilon=\exp \left\{\frac{\beta^{2}}{\pi \epsilon^{2}}\right\}\left[1-\operatorname{erf}\left(\frac{\beta}{\sqrt{\pi} \epsilon}\right)\right],
$$

where the non-dimensional integral timescale $\epsilon$ is defined as

$$
\epsilon=\frac{\mathcal{T}}{\mathcal{T}_{\text {Frozen }}}=\frac{\mathcal{T} U_{c}}{\mathcal{L}} .
$$

Equation (92) can be solved for a given value of $\epsilon$ in the range $0<\epsilon<1$, to determine the appropriate value of $\beta$ such that $\mathcal{T}$ in the expression for $\mathcal{F}(\tau)(23)$ is consistent with the definition of the integral timescale given by Eq. 20]. 


\section{Funding Sources}

The work was funded by the Engineering and Physical Sciences Research Council Grant no. EP/K017551/1.

Also the authors wish to acknowledge the continuing support provided by Rolls-Royce plc through the University Technology Centre in Gas Turbine Noise at the Institute of Sound and Vibration Research.

\section{Acknowledgments}

The authors would like to acknowledge the contributions of the late Alexander M. Cargill who originally formulated the scattering models used in this work while he was employed at Rolls-Royce plc.

\section{References}

[1] Candel, S., Guedel, A., and Julienne, A., "Refraction and scattering of sound in an open wind tunnel flow," Proceedings of the Sixth International Congress on Instrumentation in Aerospace Simulation Facilities, Ottawa, Canada, September 22-24, 1975, pp. $288-300$.

[2] Ahuja, K., Tester, B., and Tanna, H., "The free jet as a simulator of forward velocity effects on jet noise," NASA Contract Report 3056, 1978.

[3] Campos, L., "The spectral broadening of sound by turbulent shear layers. Part 1. The transmission of sound through turbulent shear layers," Journal of Fluid Mechanics, Vol. 89, 1978, pp. 723-749.

[4] Campos, L., "The spectral broadening of sound by turbulent shear layers. Part 2. The spectral broadening of sound and aircraft noise," Journal of Fluid Mechanics, Vol. 89, 1978, pp. 751-783.

[5] Guedel, A., "Scattering of an acoustic field by a free jet shear layer," Journal of Sound and Vibration, Vol. 100, No. 2, 1985, pp. 285-304.

[6] Powles, C., Tester, B., and McAlpine, A., "A weak-scattering model for turbine-tone haystacking outside the cone of silence," International Journal of Aeroacoustics, Vol. 10, No. 1, 2011, pp. 17-50.

[7] McAlpine, A., Powles, C., and Tester, B., “A weak-scattering model for turbine-tone haystacking," Journal of Sound and Vibration, Vol. 332, 2013, pp. 3806-3831.

[8] Cargill, A., "Sound propagation through fluctuating flows - Its significance in aeroacoustics," AIAA paper no. 83-0697, 1983. Proceedings of the 8th AIAA Aeroacoustics Conference, Atlanta, Georgia, April 11-13, 13 pp. 
[9] Ewert, R., Kornow, O., Tester, B., Powles, C., Delfs, J., and Rose, M., “Spectral broadening of jet engine turbine tones,” AIAA paper no. 2008-2940, 2008. Proceedings of the 14th AIAA/CEAS Aeroacoustics Conference, Vancouver, Canada, May 5-7.

[10] Ewert, R., Kornow, O., Delfs, J., Yin, J., Rober, T., and Rose, M., “A CAA based approach to tone haystacking,” AIAA 2009-3217, 2009. Proceedings of the 15th AIAA/CEAS Aeroacoustics Conference, Miami, Florida, May 11-13, 30 pages.

[11] Clair, V., and Gabard, G., "Numerical assessment of the scattering of acoustic waves by turbulent structures," AIAA 2015-2680, 2015. Proceedings of the 21st AIAA/CEAS Aeroacoustics Conference, Dallas, Texas, June 22-26, 23 pages.

[12] Clair, V., and Gabard, G., "Spectral broadening of acoustic waves by convected vortices," Journal of Fluid Mechanics, Vol. 841,2018 , pp. 50-80.

[13] Clair, V., and Gabard, G., "Numerical investigation on the spectral broadening of acoustic waves by a turbulent layer," $A I A A$ 2016-2701, 2016. Proceedings of the 22nd AIAA/CEAS Aeroacoustics Conference, Lyon, France, 30 May - 1 June, 14 pages.

[14] Bennaceur, I., Mincu, D., Mary, I., Terracol, M., Larcheveque, L., and Dupont, P., "Numerical simulation of acoustic scattering by a plane turbulent shear layer: Spectral broadening study," Computers and Fluids, Vol. 138, 2016, pp. 83-98.

[15] Krober, S., Hellmold, M., and Koop, L., "Experimental investigation of spectral broadening of sound waves by wind tunnel shear layers," AIAA 2013-2255, 2013. Proceedings of the 19th AIAA/CEAS Aeroacoustics Conference, Berlin, Germany, May 27-29, 15 pages.

[16] Sijtsma, P., Oerlemans, S., Tibbe, T., Berkefeld, T., and Spehr, C., "Spectral broadening by shear layers of open jet wind tunnels," AIAA Aviation, AIAA 2014-3178, 2014. Proceedings of the 20th AIAA/CEAS Aeroacoustics Conference, Atlanta, Georgia, June 16-20, 34 pages.

[17] Tester, B., and Sijtsma, P., "Measurement and analysis of phased array data on haystacked tones from a source located in a free jet," AIAA Aviation, AIAA 2017-3864, 2017. Proceedings of the 23rd AIAA/CEAS Aeroacoustics Conference, Denver, Colorado, June 5-9, 12 pages.

[18] McAlpine, A., and Tester, B., "A weak-scattering model for tone haystacking caused by sound propagation through an axisymmetric turbulent shear layer," AIAA 2016-2702, 2016. Proceedings of the 22nd AIAA/CEAS Aeroacoustics Conference, Lyon, France, May 30 - June 1, 27 pages.

[19] Goldstein, M., “An exact form of Lilley's equation with a velocity quadrupole/temperature dipole source term,” Journal of Fluid Mechanics, Vol. 443, 2001, pp. 231-236.

[20] Hussein, H., Capp, S., and George, W., "Velocity measurements in a high-Reynolds-number, momentum-conserving, axisymmetric, turbulent jet," Journal of Fluid Mechanics, Vol. 258, 1994, pp. 31-75. 
[21] Tam, C., and Auriault, L., "Jet mixing noise from fine-scale turbulence," AIAA Journal, Vol. 37, No. 2, February 1999, pp. $145-153$.

[22] Ewert, R., "Broadband slat noise predictions based on CAA and stochastic sound sources from fast random-particle mesh (RPM) method," Computers and Fluids, Vol. 37, 2008, pp. 369-387.

[23] Morris, P., and Boluriaan, S., "The prediction of jet noise from CFD data," AIAA paper no. 2004-2977, 2004. Proceedings of the 10th AIAA/CEAS Aeroacoustics Conference, Manchester, UK, May 10-12, 11 pp.

[24] Batchelor, G., The Theory of Homogeneous Turbulence, Cambridge University Press, 1953.

[25] Batchelor, G., "The theory of axisymmetric turbulence," Proceedings of the Royal Society of London. Series A, Mathematical and Physical Sciences, Vol. 186, No. 1007, 1946, pp. 480-502.

[26] Chandrasekhar, S., "The theory of axisymmetric turbulence," Philosophical Transactions of the Royal Society of London. Series A, Mathematical and Physical Sciences, Vol. 242, No. 855, 1950, pp. 557-577.

[27] Linborg, E., "Kinematics of homogeneous axisymmetric turbulence," Journal of Fluid Mechanics, Vol. 302, 1995 , pp. $179-201$.

[28] Townsend, A., The Structure of Turbulent Shear Flow, $2^{\text {nd }}$ ed., Cambridge University Press, 1976. 